\title{
New class of ghost- and tachyon-free metric affine gravities
}

\author{
R. Percacci $\oplus^{1}$ and E. Sezgin $\oplus^{2}$ \\ ${ }^{1}$ SISSA, via Bonomea 265, 34136 Trieste, Italy and INFN, Sezione di Trieste \\ ${ }^{2}$ George and Cynthia Woods Mitchell Institute for Fundamental Physics and Astronomy, \\ Texas A\&M University, College Station, Texas 77843, USA
}

(Received 22 January 2020; accepted 20 March 2020; published 20 April 2020)

\begin{abstract}
We construct the spin-projection operators for a theory containing a symmetric two-index tensor and a general three-index tensor. We then use them to analyze, at linearized level, the most general action for a metric affine theory of gravity with terms up to second order in curvature, which depends on 28 parameters. In the metric case, we recover known results. In the torsion-free case, we are able to determine the most general six-parameter class of theories that are projective invariant, contain only one massless spin 2 and no spin 3, and are free of ghosts and tachyons.
\end{abstract}

DOI: 10.1103/PhysRevD.101.084040

\section{INTRODUCTION}

Metric-affine gravity (MAG) is a broad class of theories of gravity based on an independent metric (or tetrad) and connection. The study of MAG has a long history [1,2]. A general linear connection will have torsion and nonmetricity. In the literature, more attention has been given to theories with torsion, but recently, there has been a great deal of interest for MAGs with nonmetricity; see, e.g., Refs. [3-13].

There can be many reasons to study such theories. The main reason for our interest in MAG is its relation to quadratic gravity ${ }^{1}$ and its similarity to gauge theories of the fundamental interactions. Quadratic gravity is known to be renormalizable [14] and asymptotically free [15] but prima facie not unitary, as expected of a theory with a kinetic term with four derivatives. There have been many proposals to circumvent this problem, but none has proven entirely convincing [16-19]. More recent progress has been reported in Refs. [20-22]. In spite of this, there has been a revival of interest in quadratic gravity, especially in connection with the possibility of realizing scale invariance at high energy [23-27].

MAG is closely related to quadratic gravity, since it can be rewritten as quadratic gravity coupled to a specific matter type. Let $A$ denote a general linear connection and $F$ denote its curvature; also, let $\Gamma$ be the Levi-Civita

\footnotetext{
${ }^{1}$ By quadratic gravity, we mean theories with action containing terms linear and quadratic in the Riemann tensor.

Published by the American Physical Society under the terms of the Creative Commons Attribution 4.0 International license. Further distribution of this work must maintain attribution to the author(s) and the published article's title, journal citation, and DOI. Funded by SCOAP ${ }^{3}$.
}

connection and $R$ be its curvature. Splitting $A=\Gamma+\phi$, where $\phi$ is a general three-index tensor, an action of the form $\int\left(F+F^{2}\right)$ becomes, schematically,

$$
\int\left[R+\phi^{2}+\left(R+\nabla \phi+\phi^{2}\right)^{2}\right]
$$

In this way, one can study large classes of theories of gravity and matter with special geometrical features. ${ }^{2}$ In MAG, the kinetic terms contain only two derivatives, but ghosts are still generically present, due to the indefiniteness of the quadratic form $F^{2}$. Thus, much of the discussion that is going on for quadratic gravity could be applied also to MAG. However, the status of MAG is much less understood.

It is thus of obvious interest to determine what special classes of MAGs could be free of ghosts and tachyons. In the metric case, the most general ghost and tachyon-free theories not containing accidental symmetries ${ }^{3}$ have been determined in Refs. [29,30]. It was based on the use of spin projectors for a general two-index tensor and a three-index tensor, antisymmetric in one pair. ${ }^{4}$ This has been extended to include parity-violating terms [31,32], and a more detailed analysis of a large number of cases including also accidental symmetries has been given recently in Ref. [33]. A broader analysis of the spectrum of a Poincaré gauge theory has been given in Ref. [34], in which a class of

\footnotetext{
${ }^{2}$ As an example, let us mention here Weyl geometry, in which $\phi$ is constructed in terms of a vector field. This theory has been revisited recently in Ref. [28].

${ }^{3}$ By accidental symmetry, we mean a gauge symmetry that is present in the linearized action but not in the full action.

${ }^{4}$ This is due to the use of the vierbein formalism. The general two-index tensor is the linearized vierbein, and the three-index tensor is the linearized spin connection.
} 
ghost- and tachyon-free models was obtained. The purpose of this paper is to give the tools that are necessary to address this problem for general MAG, containing both torsion and nonmetricity, and to exhibit a new class of ghost- and tachyon-free theories with nonmetricity.

The relation of MAG to gauge theories of fundamental interactions is best understood if one uses arbitrary frames in the tangent bundle. The theory is then seen to have a local gauge invariance under diffeomorphisms and under local $G L(4)$ transformations, but it is in a Higgs phase [35-38]. The frame field, the metric, and the connection are all independent, with the first two playing the role of Goldstone bosons. The gauge $G L(4)$ is "spontaneously broken" to the trivial group, and the connection (or more precisely the difference between the connection and the Levi-Civita connection) becomes massive.

This formalism is not well suited for practical applications because it contains a large number of redundant fields (essentially, the 16 components of the frame field). In a linearized analysis, one would discover that these fields are all part of the kernel of the kinetic operator and can be gauge fixed to be zero. It is convenient instead to work from the start with a formalism that contains the smallest number of fields. This is the standard formulation in terms of a metric $g_{\mu \nu}$ and an independent connection $A_{\lambda}{ }^{\mu}{ }_{\nu}$. In this formalism, the only gauge freedom is the diffeomorphism group, and one cannot reduce the number of fields further while preserving locality. ${ }^{5}$ It is important, however, to keep in mind that this is just a gauge-fixed version of the general

$G L(4)$ formulation and is gauge equivalent to the vierbein formulation.

In the following, we start from the most general MAG action which contains 28 free parameters and determine the conditions under which it has additional symmetries under shifts of the connection. We then determine the spin projection operators for the fields that appear in the linearized action, which facilitate the inversion of the wave operator to obtain the propagator for each spin sector. We then specialize these results to the case of theories with metric or torsion-free connections. In the latter case, we determine a six-parameter family of theories that are ghost and tachyon free, propagating a massless graviton and massive spin- $2^{-},-1^{+}$, and $-1^{-}$states with distinct masses.

\section{METRIC AFFINE GRAVITY}

\section{A. Action}

In the model we shall consider, the independent dynamical variables are the metric $g_{\mu \nu}$ of signature $-+\cdots+$ and a linear connection $A_{\mu}{ }^{\rho} \sigma$ The curvature is defined as

$$
F_{\mu \nu}{ }^{\rho}{ }_{\sigma}=\partial_{\mu} A_{\nu}{ }^{\rho}{ }_{\sigma}-\partial_{\nu} A_{\mu}{ }^{\rho}{ }_{\sigma}+A_{\mu}{ }^{\rho}{ }_{\tau} A_{\nu}{ }^{\tau}{ }_{\sigma}-A_{\nu}{ }^{\rho}{ }_{\tau} A_{\mu}{ }^{\tau}{ }_{\sigma},
$$

whereas torsion and nonmetricity are defined by ${ }^{6}$

$$
\begin{gathered}
T_{\mu^{\alpha}{ }_{\nu}{ }^{2}=A_{\mu}{ }^{\alpha}{ }_{\nu}-A_{\nu}{ }^{\alpha},}, \\
Q_{\lambda \mu \nu}=-\partial_{\lambda} g_{\mu \nu}+A_{\lambda}{ }^{\tau}{ }_{\mu} g_{\tau \nu}+A_{\lambda}{ }^{\tau}{ }_{\nu} g_{\mu \tau} .
\end{gathered}
$$

For an action, we take

$$
\begin{aligned}
S(g, A)= & -\frac{1}{2} \int d^{d} x \sqrt{|g|}\left[-a_{0} F+F^{\mu \nu \rho \sigma}\left(c_{1} F_{\mu \nu \rho \sigma}+c_{2} F_{\mu \nu \sigma \rho}+c_{3} F_{\rho \sigma \mu \nu}+c_{4} F_{\mu \rho \nu \sigma}\right.\right. \\
& \left.+c_{5} F_{\mu \sigma \nu \rho}+c_{6} F_{\mu \sigma \rho \nu}\right)+F^{(13) \mu \nu}\left(c_{7} F_{\mu \nu}^{(13)}+c_{8} F_{\nu \mu}^{(13)}\right)+F^{(14) \mu \nu}\left(c_{9} F_{\mu \nu}^{(14)}+c_{10} F_{\nu \mu}^{(14)}\right) \\
& +F^{(14) \mu \nu}\left(c_{11} F_{\mu \nu}^{(13)}+c_{12} F_{\nu \mu}^{(13)}\right)+F^{\mu \nu}\left(c_{13} F_{\mu \nu}+c_{14} F_{\mu \nu}^{(13)}+c_{15} F_{\mu \nu}^{(14)}\right)+c_{16} F^{2} \\
& +T^{\mu \rho \nu}\left(a_{1} T_{\mu \rho \nu}+a_{2} T_{\mu \nu \rho}\right)+a_{3} T^{\mu} T_{\mu}+Q^{\rho \mu \nu}\left(a_{4} Q_{\rho \mu \nu}+a_{5} Q_{\nu \mu \rho}\right) \\
& \left.+a_{6} Q^{\mu} Q_{\mu}+a_{7} \tilde{Q}^{\mu} \tilde{Q}_{\mu}+a_{8} Q^{\mu} \tilde{Q}_{\mu}+a_{9} T^{\mu \rho \nu} Q_{\mu \rho \nu}+T^{\mu}\left(a_{10} Q_{\mu}+a_{11} \tilde{Q}_{\mu}\right)\right]
\end{aligned}
$$

where

$$
\begin{array}{rlrl}
T_{\mu}:=T_{\lambda \mu}^{\lambda}, & Q_{\mu}:=Q_{\mu \lambda}{ }^{\lambda}, & \tilde{Q}_{\mu}:=Q_{\lambda \mu}^{\lambda}, \\
F_{\mu \nu}:=F_{\mu \nu \lambda^{\lambda}}, & F_{\mu \nu}^{(14)}:=F_{\lambda \mu \nu}{ }^{\lambda}, & F_{\mu \nu}^{(13)}:=F_{\lambda \mu}{ }^{\lambda}, & F:=F_{\mu \nu}{ }^{\mu \nu} .
\end{array}
$$

Note that there are two "pseudo-Ricci" tensors $F_{\mu \nu}^{(13)}$ and $F_{\mu \nu}^{(14)}$, without symmetry properties, and one pseudo-Ricci scalar that we denote the $a_{0} F$ term. The Einstein-Hilbert action is described by the $a_{0} g^{\mu \nu} F_{\mu \nu}^{(13)}$ term. The action contains 28 parameters, namely, $\left(a_{0}, a_{1}, \ldots, a_{11}, c_{1}, \ldots, c_{16}\right)$. In $d=4$, however, the combination

\footnotetext{
${ }^{5}$ Except for the possible choice of unimodular gauge; see Ref. [39].

${ }^{6}$ Note that the torsion tensor is antisymmetric in its first and third indices. This is not to be confused with the convention used widely in the supergravity literature in which it is antisymmetric in its first two indices instead.
} 


$$
\begin{aligned}
& F_{\mu \nu \rho \sigma} F^{\rho \sigma \mu \nu}-F_{\mu \nu}^{(13)} F^{\nu \mu(13)}-F_{\mu \nu}^{(14)} F^{(14) \nu \mu} \\
& \quad+2 F_{\mu \nu}^{(13)} F^{\nu \mu(14)}+F^{2}
\end{aligned}
$$

which reduces to the Gauss-Bonnet integrand in the Riemannian case, does not contribute at quadratic level when expanding around flat space. Indeed, in Weyl geometry (i.e., if the nonmetricity is of the form $Q_{\lambda \mu \nu}=v_{\lambda} g_{\mu \nu}$ ), it is a total derivative [40]. In the presence of trace-free nonmetricity, it is not a total derivative [41], but in flat space, it only gives cubic and quartic interactions. Thus, for the purposes of our analysis, one parameter is redundant. Turning to the action (2.4), it is convenient to express it as

$$
\begin{aligned}
S(g, A)= & -\frac{1}{2} \int d^{d} x \sqrt{|g|}\left[G^{\mu_{1} \ldots \mu_{4}, \nu_{1} \ldots \nu_{4}} F_{\mu_{1} \ldots \mu_{4}} F_{\nu_{1} \ldots \nu_{4}}\right. \\
& +A^{\mu_{1} \mu_{2} \mu_{3}, \nu_{1} \nu_{2} \nu_{3}} T_{\mu_{1} \mu_{2} \mu_{3}} T_{\nu_{1} \nu_{2} \nu_{3}} \\
& +B^{\mu_{1} \mu_{2} \mu_{3}, \nu_{1} \nu_{2} \nu_{3}} Q_{\mu_{1} \mu_{2} \mu_{3}} Q_{\nu_{1} \nu_{2} \nu_{3}} \\
& \left.+C^{\mu_{1} \mu_{2} \mu_{3}, \nu_{1} \nu_{2} \nu_{3}} T_{\mu_{1} \mu_{2} \mu_{3}} Q_{\nu_{1} \nu_{2} \nu_{3}}\right] .
\end{aligned}
$$

The tensors $G, A, B$, and $C$ inherit the symmetries of the objects they are contracted with. Furthermore, $G, A$, and $B$ are also symmetric under the interchange of the first half of indices with the second half. In the following expressions, symmetrizations that are not already manifest are indicated ${ }^{7}$ :

$$
\begin{aligned}
& G_{\mu_{1} \ldots \mu_{4}}^{\nu_{1} \ldots \nu_{4}}=\left[\delta_{\mu_{1}}^{\nu_{1}} \delta_{\mu_{2}}^{\nu_{2}}\left(c_{1} \delta_{\mu_{3}}^{\nu_{3}} \delta_{\mu_{4}}^{\nu_{4}}+c_{2} \delta_{\mu_{3}}^{\nu_{4}} \delta_{\mu_{4}}^{\nu_{3}}\right)+c_{3} \delta_{\mu_{1}}^{\nu_{3}} \delta_{\mu_{2}}^{\nu_{4}} \delta_{\mu_{3}}^{\nu_{1}} \delta_{\mu_{4}}^{\nu_{2}}+c_{4} \delta_{\mu_{1}}^{\nu_{1}} \delta_{\mu_{2}}^{\nu_{3}} \delta_{\mu_{3}}^{\nu_{2}} \delta_{\mu_{4}}^{\nu_{4}}\right. \\
& +\delta_{\mu_{1}}^{\nu_{1}} \delta_{\mu_{2}}^{\nu_{4}}\left(c_{5} \delta_{\mu_{3}}^{\nu_{2}} \delta_{\mu_{4}}^{\nu_{3}}+c_{6} \delta_{\mu_{3}}^{\nu_{3}} \delta_{\mu_{4}}^{\nu_{2}}\right)+\eta_{\mu_{1} \mu_{3}} \eta^{\nu_{1} \nu_{3}}\left(c_{7} \delta_{\mu_{2}}^{\nu_{2}} \delta_{\mu_{4}}^{\nu_{4}}+c_{8} \delta_{\mu_{2}}^{\nu_{4}} \delta_{\mu_{4}}^{\nu_{2}}\right) \\
& +\eta_{\mu_{1} \mu_{4}} \eta^{\nu_{1} \nu_{4}}\left(c_{9} \delta_{\mu_{2}}^{\nu_{2}} \delta_{\mu_{3}}^{\nu_{3}}+c_{10} \delta_{\mu_{2}}^{\nu_{3}} \delta_{\mu_{3}}^{\nu_{2}}\right)+\eta_{\mu_{1} \mu_{4}} \eta^{\nu_{1} \nu_{3}}\left(c_{11} \delta_{\mu_{2}}^{\nu_{2}} \delta_{\mu_{3}}^{\nu_{4}}+c_{12} \delta_{\mu_{2}}^{\nu_{4}} \delta_{\mu_{3}}^{\nu_{2}}\right) \\
& +\eta_{\mu_{3} \mu_{4}}\left(c_{13} \eta^{\nu_{3} \nu_{4}} \delta_{\mu_{1}}^{\nu_{1}} \delta_{\mu_{2}}^{\nu_{2}}+c_{14} \eta^{\nu_{1} \nu_{3}} \delta_{\mu_{1}}^{\nu_{2}} \delta_{\mu_{2}}^{\nu_{4}}+c_{15} \eta^{\nu_{1} \nu_{4}} \delta_{\mu_{1}}^{\nu_{2}} \delta_{\mu_{2}}^{\nu_{3}}\right) \\
& \left.+c_{16} \eta_{\mu_{1} \mu_{3}} \eta_{\mu_{2} \mu_{4}} \eta^{\nu_{1} \nu_{3}} \eta^{\nu_{2} \nu_{4}}\right]_{\left[\mu_{1} \mu_{2}\right]\left[\nu_{1} \nu_{2}\right]} \text {, } \\
& A_{\mu_{1} \mu_{2} \mu_{3}}{ }_{1} \nu_{2} \nu_{3}=\left[\delta_{\mu_{1}}^{\nu_{1}}\left(a_{1} \delta_{\mu_{2}}^{\nu_{2}} \delta_{\mu_{3}}^{\nu_{3}}+a_{2} \delta_{\mu_{2}}^{\nu_{3}} \delta_{\mu_{3}}^{\nu_{2}}\right)+a_{3} \eta_{\mu_{1} \mu_{2}} \eta^{\nu_{1} \nu_{2}} \delta_{\mu_{3}}^{\nu_{3}}\right]_{\left[\mu_{1} \mu_{3}\right]\left[\nu_{1} \nu_{3}\right]}, \\
& B_{\mu_{1} \mu_{2} \mu_{3}}{ }_{\nu_{1} \nu_{2} \nu_{3}}=\left[a_{4} \delta_{\mu_{1}}^{\nu_{1}} \delta_{\mu_{2}}^{\nu_{2}} \delta_{\mu_{3}}^{\nu_{3}}+a_{5} \delta_{\mu_{1}}^{\nu_{3}} \delta_{\mu_{2}}^{\nu_{2}} \delta_{\mu_{3}}^{\nu_{1}}+a_{6} \eta_{\mu_{2} \mu_{3}} \eta^{\nu_{2} \nu_{3}} \delta_{\mu_{1}}^{\nu_{1}}\right. \\
& \left.+\eta_{\mu_{1} \mu_{2}}\left(a_{7} \eta^{\nu_{1} \nu_{2}} \delta_{\mu_{3}}^{\nu_{3}}+a_{8} \eta^{\nu_{2} \nu_{3}} \delta_{\mu_{3}}^{\nu_{1}}\right)\right]_{\left(\mu_{2} \mu_{3}\right)\left(\nu_{2} \nu_{3}\right)}, \\
& C_{\mu_{1} \mu_{2} \mu_{3}}{ }_{1}^{\nu_{2} \nu_{2} \nu_{3}}=\left[a_{9} \delta_{\mu_{1}}^{\nu_{1}} \delta_{\mu_{2}}^{\nu_{2}} \delta_{\mu_{3}}^{\nu_{3}}+\eta^{\nu_{1} \nu_{2}}\left(a_{10} \eta_{\mu_{2} \mu_{3}} \delta_{\mu_{1}}^{\nu_{3}}+a_{11} \eta_{\mu_{1} \mu_{2}} \delta_{\mu_{3}}^{\nu_{3}}\right)\right]_{\left[\mu_{1} \mu_{3}\right]\left(\nu_{2} \nu_{3}\right)},
\end{aligned}
$$

where it is understood that $G$ is to be symmetrized with respect to interchange of indices $\left(\mu_{1} \ldots \mu_{4}\right)$ and $\left(\nu_{1} \ldots \nu_{4}\right)$ and that $A, B$, and $C$ are to be symmetrized with respect to the interchange of indices $\left(\mu_{1} \ldots \mu_{3}\right)$ and $\left(\nu_{1} \ldots \nu_{3}\right)$.

\section{B. Gauge symmetries}

In general, the action is invariant under the action of diffeomorphisms,

$$
\begin{gathered}
g_{\mu \nu}^{\prime}\left(x^{\prime}\right)=\frac{\partial x^{\alpha}}{\partial x^{\prime \mu}} \frac{\partial x^{\beta}}{\partial x^{\prime \nu}} g_{\alpha \beta}(x), \\
A_{\mu \beta}^{\prime \alpha}\left(x^{\prime}\right)=\frac{\partial x^{\nu}}{\partial x^{\prime \mu}} \frac{\partial x^{\prime \alpha}}{\partial x^{\gamma}} \frac{\partial x^{\delta}}{\partial x^{\prime \beta}} A_{\nu}{ }^{\gamma} \delta(x)+\frac{\partial x^{\prime \alpha}}{\partial x^{\gamma}} \frac{\partial^{2} x^{\gamma}}{\partial x^{\prime \mu} \partial x^{\prime \beta}} .
\end{gathered}
$$

For an infinitesimal transformation $x^{\prime \mu}=x^{\mu}-\xi^{\mu}(x)$, the transformation is given by the Lie derivatives, plus an inhomogeneous term for the connection,

\footnotetext{
${ }^{7}$ In our conventions, the (anti)symmetrizations are always with unit strength, e.g., $X_{[a} Y_{b]}=\frac{1}{2}\left(X_{a} Y_{b}-X_{b} Y_{a}\right)$.
}

$$
\delta g_{\mu \nu}=\mathcal{L}_{\xi} g_{\mu \nu}, \delta A_{\rho^{\mu}}{ }_{\nu}=\mathcal{L}_{\xi} A_{\rho}{ }^{\mu}{ }_{\nu}+\partial_{\rho} \partial_{\nu} \xi^{\mu},
$$

where $\mathcal{L}_{\xi} A_{\rho}{ }^{\mu}{ }_{\nu}=\xi^{\lambda} \partial_{\lambda} A_{\rho}{ }^{\mu}{ }_{\nu}+A_{\lambda}{ }^{\mu}{ }_{\nu} \partial_{\rho} \xi^{\lambda}-A_{\rho}{ }^{\lambda}{ }_{\nu} \partial_{\lambda} \xi^{\mu}+A_{\rho}{ }^{\mu}{ }_{\lambda} \partial_{\nu} \xi^{\lambda}$. In four dimensions, if all the coefficients $a_{i}$ are zero, the action is additionally invariant under the following realization of Weyl transformations:

$$
\delta g_{\mu \nu}=2 \omega g_{\mu \nu} ; \quad \delta A_{\mu}{ }^{\rho}{ }_{\nu}=0 .
$$

This is the usual way in which Weyl transformations are realized on Yang-Mills fields, while the Levi-Civita connection transforms as

$$
\delta \Gamma_{\mu}{ }^{\rho}{ }_{\nu}=\partial_{\mu} \omega \delta_{\nu}^{\rho}+\partial_{\nu} \omega \delta_{\mu}^{\rho}-g^{\rho \tau} \partial_{\tau} \omega g_{\mu \nu} .
$$

In the following, we shall be interested in cases in which the action is invariant under additional transformations of the connection (see also Ref. [42]). The following three classes of transformations will be relevant. First, we consider the projective transformations

$$
\delta_{1} A_{\mu}{ }^{\rho}{ }_{\nu}=\lambda_{\mu} \delta_{\nu}^{\rho}, \quad \delta_{1} g_{\mu \nu}=0
$$


where $\lambda_{\mu}(x)$ is an arbitrary gauge parameter. Under this transformation,

$$
\begin{aligned}
\delta_{1} F_{\mu \nu \rho \sigma} & =\left(2 \nabla_{[\mu} \lambda_{\nu]}+T_{\mu}{ }^{\tau}{ }_{\nu} \lambda_{\tau}\right) g_{\rho \sigma}=2 \partial_{[\mu} \lambda_{\nu]} g_{\rho \sigma}, \\
\delta_{1} T_{\mu}{ }^{\rho}{ }_{\nu} & =2 \lambda_{[\mu} \delta_{\nu]}^{\rho}, \quad \delta_{1} Q_{\rho \mu \nu}=2 \lambda_{\rho} g_{\mu \nu} .
\end{aligned}
$$

In particular, $\delta_{1} F=0$. Assuming that neither torsion nor the nonmetricity vanish, one finds that the action is invariant, provided that

$$
\begin{aligned}
2 c_{1}+2 c_{2}+2 d c_{13}-c_{14}-c_{15} & =0, \\
c_{5}+2 c_{6}+2 c_{7}-2 c_{8}+c_{11}-c_{12}-d c_{14} & =0, \\
2 c_{4}+c_{5}+2 c_{9}-2 c_{10}+c_{11}-c_{12}-d c_{15} & =0, \\
2 a_{1}+a_{2}+(d-1) a_{3}+a_{9}-d a_{10}-a_{11} & =0, \\
4 a_{4}+4 d a_{6}+2 a_{8}+a_{9}-(d-1) a_{10} & =0, \\
4 a_{5}+4 a_{7}+2 d a_{8}-a_{9}-(d-1) a_{11} & =0 .
\end{aligned}
$$

There is a similar transformation with the second index singled out

$$
\delta_{2} A_{\mu}{ }^{\rho}{ }_{\nu}=\lambda^{\rho} g_{\mu \nu}, \quad \delta_{2} g_{\mu \nu}=0,
$$

under which

$\delta_{2} F_{\mu \nu \rho \sigma}=2 g_{\sigma[\nu} \nabla_{\mu]} \lambda_{\rho}+2 g_{\sigma[\nu} Q_{\mu] \rho \tau} \lambda^{\tau}+2\left(Q_{[\nu \mu] \sigma}+T_{\mu \sigma \nu}\right) \lambda_{\rho}$,

$\delta_{2} T_{\mu}{ }^{\rho}{ }_{\nu}=0, \quad \delta_{2} Q_{\rho \mu \nu}=2 g_{\rho(\mu} \lambda_{\nu}$.
In this case, the variation of the general action gives rise to a large number of independent structures. Then, the invariance of the action requires that

$$
\begin{array}{r}
c_{1}=c_{2}=\ldots=c_{16}=0, \\
(2-d) a_{0}+a_{9}+2 a_{10}+(d+1) a_{11}=0, \\
(3-d) a_{0}+4 a_{5}+8 a_{6}+2(d+1) a_{8}=0, \\
-a_{0}+4 a_{4}+2 a_{5}+2(d+1) a_{7}+2 a_{8}=0 .
\end{array}
$$

Finally, there is the transformation that singles out the third index

$$
\delta_{3} A_{\mu}{ }^{\rho}{ }_{\nu}=\delta_{\mu}^{\rho} \lambda_{\nu}, \quad \delta g_{\mu \nu}=0,
$$

under which

$$
\begin{aligned}
\delta_{3} F_{\mu \nu \rho \sigma} & =2 g_{\rho[\nu} \nabla_{\mu]} \lambda_{\sigma}+T_{\mu \rho \nu} \lambda_{\sigma}, \\
\delta_{3} T_{\mu}{ }^{\rho}{ }_{\nu} & \left.=2 \delta_{[\mu}^{\rho} \lambda_{\nu]}, \quad \delta_{3} Q_{\rho \mu \nu}=2 g_{\rho(\mu} \lambda_{\nu}\right)
\end{aligned}
$$

Once again, the variation of the general action gives rise to a large number of independent structures. Assuming that the torsion and nonmetricity do not vanish, the action is invariant, provided that

$$
\begin{array}{r}
c_{1}=c_{2}=\cdots=c_{16}=0, \\
(d-2) a_{0}+4 a_{1}+2 a_{2}+2(d-1) a_{3}+a_{9}+2 a_{10}+(d+1) a_{11}=0, \\
(d-1) a_{0}+4 a_{5}+8 a_{6}+2(d+1) a_{8}-2 a_{9}+2(d-1) a_{10}=0, \\
(1-d) a_{0}+4 a_{4}+2 a_{5}+2(d+1) a_{7}+2 a_{8}+a_{9}+(d-1) a_{11}=0 .
\end{array}
$$

\section{LINEARIZATION AND SPIN PROJECTORS}

\section{A. Linearized action}

The equations of motion that come from the action (2.4) have as a solution the Minkowski space

$$
g_{\mu \nu}=\eta_{\mu \nu}, \quad A_{\rho_{\nu}}{ }_{\nu}=0 .
$$

Expanding the action around this solution, the quadratic wave operator takes the form

$$
\begin{aligned}
S^{(2)}= & \frac{1}{2} \int d^{d} q\left(A^{\lambda \mu \nu} \mathcal{O}_{\lambda \mu \nu}{ }^{\tau \rho \sigma} A_{\tau \rho \sigma}+2 A^{\lambda \mu \nu} \mathcal{O}_{\lambda \mu \nu}{ }^{\rho \sigma} h_{\rho \sigma}\right. \\
& \left.+h^{\mu \nu} \mathcal{O}_{\mu \nu}{ }^{\rho \sigma} h_{\rho \sigma}\right),
\end{aligned}
$$

where, by abuse of notation, we denote $A$ also the fluctuation and

$$
\begin{aligned}
\mathcal{O}^{\mu \nu, \rho \sigma}= & -B^{\lambda \mu \nu, \tau \rho \sigma} q_{\lambda} q_{\tau}, \\
\mathcal{O}^{\lambda \mu \nu, \rho \sigma}= & -2 i\left(A^{\lambda \mu \nu, \tau \rho \sigma}+C^{\lambda \mu \nu, \tau \rho \sigma}\right) q_{\tau} \\
& +\frac{i}{2} a_{0}\left[\eta^{\nu \sigma}\left(\eta^{\lambda \rho} q^{\mu}-\eta^{\lambda \mu} q^{\rho}\right)-\frac{1}{2} \eta^{\rho \sigma}\left(\eta^{\lambda \nu} q^{\mu}-\eta^{\lambda \mu} q^{\nu}\right)\right], \\
\mathcal{O}^{\lambda \mu \nu, \tau \rho \sigma}= & -4\left(G^{\kappa \lambda \mu \nu, \eta \tau \rho \sigma} q_{\kappa} q_{\eta}+A^{\lambda \mu \nu, \tau \rho \sigma}+B^{\lambda \mu \nu, \tau \rho \sigma}\right. \\
& \left.+2 C^{\lambda \mu \nu, \tau \rho \sigma}\right)+a_{0} \eta^{\nu \rho}\left(\eta^{\lambda \mu} \eta^{\tau \sigma}-\eta^{\lambda \sigma} \eta^{\mu \tau}\right) .
\end{aligned}
$$

This operator has a kernel consisting (at least) of the infinitesimal diffeomorphisms (2.14), which in the present case read

$$
\delta g_{\mu \nu}=\partial_{\mu} \xi_{\nu}+\partial_{\nu} \xi_{\mu}, \quad \delta A_{\lambda \mu \nu}=\partial_{\nu} \partial_{\lambda} \xi_{\mu}
$$

For specific values of the couplings, the kernel could be larger. 


\section{B. Spin projectors}

In the analysis of the spectrum of operators acting on multi-index fields in flat space, it is very convenient to use spin-projection operators, which can be used to decompose the fields in their irreducible components under the threedimensional rotation group [43-45]. For a three-index tensor that is antisymmetric in one pair of indices, the spin projectors were given in Refs. $[29,46]$. The spin projectors for totally symmetric three-index tensors have been given also in Ref. [47]. To the best of our knowledge, the spin projectors for a general three-index tensor have not been given in the literature. We thus turn to the construction of these objects.

\section{1. $G L(d)$ decomposition}

The space of two-index tensors can be decomposed into irreducible representations (irreps) of the group $G L(d)$, given by symmetric and antisymmetric tensors. The projectors onto these subspaces are

$$
\Pi_{a b}^{(s / a) e f}=\frac{1}{2}\left(\delta_{a}^{e} \delta_{b}^{f} \pm \delta_{a}^{f} \delta_{b}^{e}\right) .
$$

The finer decomposition into irreps of $S O(d-1)$ is widely used in gravity. The corresponding treatment of three-index tensors is algebraically more complicated. We begin with some elementary facts about three-index tensors as representations of $G L(d)$. To discuss their symmetry properties, we will focus on the second pair of indices. Thus, when we say that $t_{c a b}$ is (anti)symmetric, without further specification, we mean $t_{c b a}=\mp t_{c a b}$.

The space $V$ of three-index tensors has dimension $d^{3}$. The subspaces $V^{(s)}$ and $V^{(a)}$ of symmetric and antisymmetric tensors are invariant subspaces of dimensions $d^{2}(d+1) / 2$ and $d^{2}(d-1) / 2$, respectively. The projectors onto these subspaces are

$$
\begin{aligned}
& \Pi_{c a b}^{(s) d e f}=\frac{1}{2} \delta_{c}^{d}\left(\delta_{a}^{e} \delta_{b}^{f}+\delta_{a}^{f} \delta_{b}^{e}\right), \\
& \Pi_{c a b}^{(a) d e f}=\frac{1}{2} \delta_{c}^{d}\left(\delta_{a}^{e} \delta_{b}^{f}-\delta_{a}^{f} \delta_{b}^{e}\right) .
\end{aligned}
$$

The subspaces $V^{(t s)}$ and $V^{(t a)}$ of totally symmetric and totally antisymmetric tensors are invariant subspaces of dimensions $d(d-1)(d-2) / 6$ and $d(d+1)(d+2) / 6$, respectively. Given any tensor, one can extract its totally (anti)symmetric part by means of the projectors

$$
\begin{aligned}
\Pi_{c a b}^{(t s) d e f}= & \frac{1}{6}\left(\delta_{c}^{d} \delta_{a}^{e} \delta_{b}^{f}+\delta_{c}^{d} \delta_{a}^{f} \delta_{b}^{e}+\delta_{c}^{f} \delta_{a}^{d} \delta_{b}^{e}+\delta_{c}^{f} \delta_{a}^{e} \delta_{b}^{d}\right. \\
& \left.+\delta_{c}^{e} \delta_{a}^{f} \delta_{b}^{d}+\delta_{c}^{e} \delta_{a}^{d} \delta_{b}^{f}\right) \\
\Pi_{c a b}^{(t a) d e f}= & \frac{1}{6}\left(\delta_{c}^{d} \delta_{a}^{e} \delta_{b}^{f}-\delta_{c}^{d} \delta_{a}^{f} \delta_{b}^{e}+\delta_{c}^{f} \delta_{a}^{d} \delta_{b}^{e}-\delta_{c}^{f} \delta_{a}^{e} \delta_{b}^{d}\right. \\
& \left.+\delta_{c}^{e} \delta_{a}^{f} \delta_{b}^{d}-\delta_{c}^{e} \delta_{a}^{d} \delta_{b}^{f}\right)
\end{aligned}
$$

The complements of $V^{(t s)}$ in $V^{(s)}$ and of $V^{(t a)}$ in $V^{(a)}$ are also invariant subspaces denoted $V^{(h s)}$ and $V^{(h a)}$, respectively. ${ }^{8}$ They consist of tensors that are (anti)symmetric but have zero totally (anti)symmetric part. The projectors onto such subspaces are

$$
\begin{aligned}
\Pi_{c a b}^{(h s) d e f} & =\Pi_{c a b}^{(s) d e f}-\Pi_{c a b}^{(t s)} d e f \\
& =\frac{1}{6}\left(2 \delta_{c}^{d} \delta_{a}^{e} \delta_{b}^{f}-\delta_{b}^{d} \delta_{c}^{e} \delta_{a}^{f}-\delta_{a}^{d} \delta_{b}^{e} \delta_{c}^{f}\right)+a \leftrightarrow b, \\
\Pi_{c a b}^{(h a) d e f} & =\Pi_{c a b}^{(a) d e f}-\Pi_{c a b}^{(t a) d e f} \\
& =\frac{1}{6}\left(2 \delta_{c}^{d} \delta_{a}^{e} \delta_{b}^{f}-\delta_{b}^{d} \delta_{c}^{e} \delta_{a}^{f}-\delta_{a}^{d} \delta_{b}^{e} \delta_{c}^{f}\right)-a \leftrightarrow b .
\end{aligned}
$$

Thus, the decomposition of a three-index tensor in its $G L(d)$-irreducible parts is

$$
t_{c a b}=t_{c a b}^{(t s)}+t_{c a b}^{(h s)}+t_{c a b}^{(h a)}+t_{c a b}^{(t a)} .
$$

where

$$
\begin{aligned}
t_{c a b}^{(t s)} & =\frac{1}{6}\left(t_{c a b}+t_{c b a}+t_{b c a}+t_{b a c}+t_{a b c}+t_{a c b}\right), \\
t_{c a b}^{(h s)} & =\frac{1}{6}\left(2 t_{c a b}+2 t_{c b a}-t_{a c b}-t_{a b c}-t_{b c a}-t_{b a c}\right), \\
t_{c a b}^{(h a)} & =\frac{1}{6}\left(2 t_{c a b}-2 t_{c b a}+t_{a c b}-t_{a b c}-t_{b c a}+t_{b a c}\right), \\
t_{c a b}^{(t a)} & =\frac{1}{6}\left(t_{c a b}-t_{c b a}+t_{b c a}-t_{b a c}+t_{a b c}-t_{a c b}\right) .
\end{aligned}
$$

\section{2. $S O(d-1)$ decomposition}

A 4-vector $q^{a}$ with $q^{2} \neq 0$ breaks $S O(1, d-1)$ to $S O(d-1)$. In physical applications, $q^{a}$ has the meaning of a timelike 4-momentum. Given $q^{a}$, we can decompose every other vector in parts longitudinal and transverse to it, by using the projectors

$\hat{q}^{a} \equiv q^{a} / \sqrt{\left|q^{2}\right|}, \quad L_{a}{ }^{b}=\hat{q}_{a} \hat{q}^{b}, \quad T_{a}{ }^{b}=\delta_{a}^{b}-L_{a}^{b}$.

This leads to a finer decomposition of $V$ into irreps of the group $S O(d-1)$. For a first step, we expand the identity

$$
\delta_{c}^{d} \delta_{a}^{e} \delta_{b}^{f}=\left(T_{c}^{d}+L_{c}^{d}\right)\left(T_{a}^{e}+L_{a}^{e}\right)\left(T_{b}^{f}+L_{b}^{f}\right)
$$

in eight terms. It is easy to see that the combinations

\footnotetext{
" " $h s "$ and " $h a "$ stand for "hook symmetric" and "hook antisymmetric," since these tensors have the structure of the hook Young tableau.
} 
TABLE I. Dimensions of projected spaces in $d$ dimensions.

\begin{tabular}{lccccc}
\hline \hline & $t s$ & $h s$ & $h a$ & $t a$ & Dimension \\
\hline$T T T$ & $\frac{d\left(d^{2}-1\right)}{6}$ & $\frac{d(d-1)(d-2)}{3}$ & $\frac{d(d-1)(d-2)}{3}$ & $\frac{(d-3)(d-2)(d-1)}{6}$ & $(d-1)^{3}$ \\
$T T L+T L T+L T T$ & $\frac{d(d-1)}{2}$ & $(d-1)^{2}$ & $(d-1)^{2}$ & $\frac{(d-2)(d-1)}{2}$ & $3(d-1)^{2}$ \\
$L L T+L T L+T L L$ & $d-1$ & $d-1$ & $d-1$ & 0 & $3(d-1)$ \\
$L L L$ & 1 & 0 & 0 & 0 & 1 \\
$\operatorname{dim}$ & $\frac{d(d+1)(d+2)}{6}$ & $\frac{d\left(d^{2}-1\right)}{3}$ & $\frac{d\left(d^{2}-1\right)}{3}$ & $\frac{d(d-1)(d-2)}{6}$ & $d^{3}$ \\
\hline \hline
\end{tabular}

$T T T ; \quad T T L+T L T+L T T$,

$T L L+L T L+L L T, \quad L L L$

(all with fixed indices) are projectors. Then, consider the simultaneous eigenspaces with eigenvalue 1 of these and of the $G L(d)$ projectors introduced above. The dimensions of these spaces are given in Table I. The last column and the last row give the total dimension of the +1 eigenspaces of the projectors in the corresponding rows and columns.

All of these spaces are representations of $S O(d-1)$, some irreducible and others not. To obtain the irreps, let us note that the $h s$ and $h a$ projections of $\frac{3}{2} L T T$ and $T T L+$ $T L T-\frac{1}{2} L T T$ are themselves projectors. Finally, in several of these representations, one can isolate the "trace" and the "trace-free" parts. In dimension $d=4$, the $S O(3)$ irreducible representations are then given in Table II, together with the spin and parity carried by them. For completeness, we also list the representations carried by the two-index symmetric tensor $h$. The subscripts refer to the number in the labeling of the projectors.

A given representation of the group $S O(3)$ may appear more than once in the decomposition of $A_{c a b}$. These copies will be distinguished by a label $i$. Thus, for example, the representation $2^{-}$occurs twice, and the two instances are denoted $2_{1}^{-}$and $2-$. In addition, the same representation may occur also in the decomposition of the 2-tensor $h_{a b}$.

TABLE II. $S O(3)$ spin content of projection operators for $A$ and $h$ in $d=4[t s / t a=$ totally (anti)symmetric; $h s / h a=$ hook (anti) symmetric].

\begin{tabular}{lcccc}
\hline \hline & $t s$ & $h s$ & $h a$ & $t a$ \\
\hline$T T T$ & $3^{-}, 1_{1}^{-}$ & $2_{1}^{-}, 1_{2}^{-}$ & $2_{2}^{-}, 1_{3}^{-}$ & $0^{-}$ \\
$T T L+T L T+L T T$ & $2_{1}^{+}, 0_{1}^{+}$ & $\ldots$ & $\cdots$ & $1_{3}^{+}$ \\
$\frac{3}{2} L T T$ & $\cdots$ & $2_{2}^{+}, 0_{2}^{+}$ & $1_{2}^{+}$, & $\cdots$ \\
$T T L+T L T-\frac{1}{2} L T T$ & $\ldots$ & $1_{1}^{+}$ & $2_{3}^{+}, 0_{3}^{+}$ & $\cdots$ \\
$T L L+L T L+L L T$ & $1_{4}^{-}$ & $1_{5}^{-}$ & $1_{6}^{-}$ & $\cdots$ \\
$L L L$ & $0_{4}^{+}$ & $\cdots$ & $\cdots$ & $\cdots$ \\
\hline \hline & & & & \multicolumn{2}{c}{$s$} \\
\hline$T T$ & & & \multicolumn{2}{c}{$2_{4}^{+}, 0_{5}^{+}$} \\
$T L$ & & & \multicolumn{2}{c}{$1_{7}^{-}$} \\
$L L$ & & & \multicolumn{2}{c}{$0_{6}^{+}$} \\
\hline \hline
\end{tabular}

We use the same label for all these representations. Thus, for example, the representation $2^{+}$occurs altogether four times: the representations $2_{i}^{+}$with $i=1,2,3$ come from $A_{c a b}$, whereas $2_{4}^{+}$comes from $h_{a b}$. The irreps carried by $A$ and $h$ are listed in Table III.

For each representation $J_{i}^{\mathcal{P}}$, there is a projector denoted $P_{i i}\left(J^{\mathcal{P}}\right)$. In addition, for each pair of representations with the same spin parity, labeled by $i, j$, there is an intertwining operator $P_{i j}\left(J^{\mathcal{P}}\right)$. We collectively refer to all the projectors and intertwiners as the "spin projectors." Formulas for all the spin projectors are given in Appendix A. For convenience, they are also given in an ancillary Mathematica notebook on the arXiv.

Let us emphasize again that these spin projectors are suitable to decompose tensors that either have no symmetry property or are (anti)symmetric in the last two indices. If one is interested in tensors that are (anti)symmetric in the first and third indices, it is more convenient to work with another set of spin projectors $P_{i j}^{\prime}\left(J^{\mathcal{P}}\right)$, such that whenever the representation $i$ or $j$ is carried by a three-index tensor the first two indices are permuted. For example,

$$
\begin{aligned}
P_{11}^{\prime}\left(2^{+}\right)^{c a b}{ }_{\text {def }} & =P_{11}\left(2^{+}\right)^{a c b_{e d f},} \\
P_{14}^{\prime}\left(2^{+}\right)^{c a b}{ }_{\text {ef }} & =P_{14}\left(2^{+}\right)^{a c b}{ }_{\text {ef }}, \text { etc. }
\end{aligned}
$$

Similarly, one can deal with tensors that are (anti)symmetric in the first two indices.

TABLE III. Count of fields of general MAG: list of irreps of given spin contained in $A$ (second column) in $h$ (third column); their total number (fourth column) and total number of fields they carry in $d=4$.

\begin{tabular}{lcccc}
\hline \hline$J^{P}$ & $A$ & $h$ & No. of irreps & No. of fields \\
\hline $3^{-}$ & 1 & $\ldots$ & 1 & 7 \\
$2^{+}$ & $1,2,3$ & 4 & 4 & 20 \\
$2^{-}$ & 1,2 & $\cdots$ & 2 & 10 \\
$1^{+}$ & $1,2,3$ & $\cdots$ & 3 & 9 \\
$1^{-}$ & $1,2,3,4,5,6$ & 7 & 7 & 21 \\
$0^{+}$ & $1,2,3,4$ & 5,6 & 6 & 6 \\
$0^{-}$ & 1 & $\cdots$ & 1 & 1 \\
total & & & & 74 \\
\hline \hline
\end{tabular}




\section{Rewriting the quadratic action}

The projector $P_{i j}\left(J^{\mathcal{P}}\right)$ has two sets of hidden indices: one for the representation $J_{i}^{\mathcal{P}}$ and one for the representation $J_{j}^{\mathcal{P}}$. These multi-indices $A, B \ldots$ consist of either three or two indices, depending whether the carrier field of the representation is $A$ or $h$. Thus, for example, $P_{11}\left(2^{+}\right)$has indices $P_{11}\left(2^{+}\right)^{c a b}{ }_{d e f}, P_{41}\left(2^{+}\right)$has indices $P_{41}\left(2^{+}\right)^{a b}{ }_{d e f}$, etc. The spin projectors satisfy the orthonormality relation

$$
P_{i j}\left(J^{\mathcal{P}}\right)^{A}{ }_{B} P_{k l}\left(I^{\mathcal{Q}}\right)^{B}{ }_{C}=\delta_{I J} \delta_{\mathcal{P} Q} \delta_{j k} \quad P_{i l}\left(J^{\mathcal{P}}\right)^{A}{ }_{C}
$$

and the completeness relation

$$
\sum_{J, \mathcal{P}, i} P_{i i}\left(J^{\mathcal{P}}\right)=\mathbf{1}
$$

The linearized quadratic action (3.3), can be written as

$$
\begin{aligned}
S^{(2)}= & \frac{1}{2} \int d^{d} q\left(\begin{array}{ll}
A(-q) & h(-q)
\end{array}\right) \\
& \times\left(\begin{array}{ll}
\mathcal{O}_{A A}(q) & \mathcal{O}_{A h}(q) \\
\mathcal{O}_{h A}(q) & \mathcal{O}_{h h}(q)
\end{array}\right)\left(\begin{array}{c}
A(q) \\
h(q)
\end{array}\right) .
\end{aligned}
$$

In four dimensions, the kinetic operator is an $74 \times 74$ matrix, that we have written as $64 \times 64,10 \times 10$ and offdiagonal $10 \times 64$ and $64 \times 10$ blocks. Since the operator is Lorentz covariant, it maps states of a given spin and parity to states of the same spin and parity. Therefore, decomposing $A_{c a b}$ and $h_{a b}$ into irreducible representations of the rotation group puts the kinetic operator in block diagonal form.

Expanding the operator $\mathcal{O}_{A B}$ in terms of these projection operators, one can rewrite the quadratic action as

$S^{(2)}=\frac{1}{2} \int d^{d} q \sum_{J P i j} \Phi(-q) \cdot a_{i j}\left(J^{\mathcal{P}}\right) P_{i j}\left(J^{\mathcal{P}}\right) \cdot \Phi(q)$.

Exploiting the relations (3.15), (3.16), the matrix elements $a_{i j}\left(J^{\mathcal{P}}\right)$, where both representations $J_{i}^{\mathcal{P}}$ and $J_{j}^{\mathcal{P}}$ are carried by $A$, can be obtained by

$$
\begin{aligned}
a_{i j}\left(J^{\mathcal{P}}\right) & =\frac{1}{d\left(J^{\mathcal{P}}\right)} P_{i j}\left(J^{\mathcal{P}}\right)^{c a b}{ }_{d e f} \mathcal{O}_{A A}^{\text {def }}{ }_{c a b} \\
& =\frac{1}{d\left(J^{\mathcal{P}}\right)} P_{k i}\left(J^{\mathcal{P}}\right)^{c a b}{ }_{d e f} \mathcal{O}_{A A}^{\text {def }}{ }_{\ell m n} P_{j k}\left(J^{\mathcal{P}}\right)^{\ell m n}{ }_{c a b},
\end{aligned}
$$

for any fixed $k$, where $d\left(J^{\mathcal{P}}\right)$ is the dimension of the representation $J^{\mathcal{P}}$. The second equality follows from (3.15), and it shows that it suffices to know the projections operators $P_{j k}$ for any fixed $k$ in order to obtain all coefficients matrices. This was also observed in
Ref. [34], in which $P_{j k}$ for a fixed $k$ (chosen for convenience to give the simplest projector) were referred to as "semiprojectors." Similarly, if the representation $J_{i}^{P}$ is carried by $A$ and $J_{j}^{\mathcal{P}}$ is carried by $h$, we can use, for example,

$$
\begin{aligned}
a_{i j}\left(J^{\mathcal{P}}\right) & =\frac{1}{d\left(J^{\mathcal{P}}\right)} P_{i j}\left(J^{\mathcal{P}}\right)^{d e}{ }_{c a b} \mathcal{O}_{A h d e}^{c a b} \\
& =\frac{1}{d\left(J^{\mathcal{P}}\right)} P_{k i}\left(J^{\mathcal{P}}\right)^{c a b}{ }_{d e f} \mathcal{O}_{A h m n}^{d e f} P_{j k}\left(J^{\mathcal{P}}\right)^{m n}{ }_{c a b},
\end{aligned}
$$

where we have chosen $k$ that is carried by $A$. These matrices $a_{i j}\left(J^{\mathcal{P}}\right)$ will be referred to as the "coefficient matrices." For a general MAG in four dimensions, they are given in Appendix B. 1.

\section{CONSTRAINTS FOR GHOST AND TACHYON FREEDOM}

Let us arrange the fluctuations into a multifield $\Phi_{A}$ and introduce corresponding sources:

$$
\Phi_{A}=\left(\begin{array}{c}
A_{c a b} \\
h_{a b}
\end{array}\right), \quad \mathcal{J}_{A}=\left(\begin{array}{c}
\tau_{c a b} \\
\sigma_{a b}
\end{array}\right) .
$$

Adding source terms, the linearized action can be written

$$
\begin{aligned}
S^{(2)}= & \int d^{d} q\left[\frac{1}{2} \sum_{J P i j} \Phi(-q) \cdot a_{i j}\left(J^{\mathcal{P}}\right) P_{i j}\left(J^{\mathcal{P}}\right) \cdot \Phi(q)\right. \\
& +\mathcal{J}(-q) \cdot \Phi(q)]
\end{aligned}
$$

which gives the field equations

$$
\sum_{J P i j} a_{i j}\left(J^{\mathcal{P}}\right) P_{i j}\left(J^{\mathcal{P}}\right) \cdot \Phi=-\mathcal{J} .
$$

Inverting for $\Phi$ as a function of $\mathcal{J}$ and substituting back into $S^{(2)}$, we obtain a quadratic form in $\mathcal{J}$ that we identify with the saturated propagator and we denote by П. There is, however, a complication: in a given spin-parity sector, the matrix $a_{i j}$ may have null eigenvectors. This corresponds to the presence of gauge symmetries as follows. Suppose for a given $J^{P}$, the matrix $a_{i j}$ is $n \times n$ and has rank $m$, thereby admitting $(n-m)$ null vectors,

$\sum_{j} a_{i j} V_{j}^{(r)}=0, \quad i, j=1, \ldots, n, \quad r=1, \ldots, n-m$.

Then, Eq. (4.2) is easily seen to be invariant under 


$$
\delta \Phi=\sum_{k, r} V_{k}^{(r)} P_{k \ell} \cdot \xi^{(r)}, \quad \forall \ell
$$

where $\xi^{(r)}$ are arbitrary functions of the coordinates, provided that the sources obey the constraints

$$
\sum_{i} V_{i}^{\dagger(r)} P_{j i} \cdot \mathcal{J}=0, \quad \forall j, r, J, \mathcal{P},
$$

The preceding analysis has to be repeated in each spin sector to determine all the gauge symmetries and source constraints. In practice, this cumbersome procedure will not be necessary for the following reasons.

Let us distinguish gauge symmetries that are already present in the original action (2.4) from "accidental" symmetries that are only present in the linearized action. The latter are broken by interactions and therefore cannot be maintained in the quantum theory. In the following, we shall restrict ourselves to theories that do not have accidental symmetries. Thus, the only infinitesimal gauge invariance is given by the diffeomorphisms (3.4):

$$
\delta A_{c a b}=-q_{b} q_{c} \xi_{a}, \quad \delta h_{a b}=i\left(q_{a} \xi_{b}+q_{b} \xi_{a}\right) .
$$

Writing this schematically as $\delta \Phi=D \xi$, since $D \xi$ is a null eigenvector of the linearized kinetic term, we must have

$$
\sum_{J P i j} a_{i j}\left(J^{\mathcal{P}}\right) P_{i j}\left(J^{\mathcal{P}}\right) D \xi=0 .
$$

Explicit calculation shows that $P_{i j}\left(J^{\mathcal{P}}\right) D \xi$ is only nonzero for $J^{\mathcal{P}}=1^{-}$and $j=4,5,6,7$ or $J^{\mathcal{P}}=0^{+}$and $j=4,6$. Then, one finds that $a\left(1^{-}\right)$has the null eigenvector

$$
(0,0,0,-i|q| / \sqrt{6},-i|q| /(2 \sqrt{3}), i|q| / 2,1),
$$

and $a\left(0^{+}\right)$has the null eigenvector $(0,0,0, i|q| / 2,0,1)$. Thus, in general, the ranks of the coefficient matrices $a\left(1^{-}\right)$ and $a\left(0^{+}\right)$are 6 and 5 , respectively. Invariance of the source term then demands that the sources satisfy the constraint ${ }^{9}$

$$
2 i q^{a} \sigma_{a c}+q^{a} q^{b} \tau_{b c a}=0 .
$$

To obtain the propagator sandwiched between physical sources, one takes the inverse of any $m \times m$ submatrix of $a_{i j}$ with nonzero determinant. This amounts to fixing the gauge symmetries, and it does not affect the form of the physical saturated propagator [48]. Denoting this submatrix by $b_{k \ell},(k, \ell=1, \ldots m)$, the resulting saturated propagator

\footnotetext{
${ }^{9}$ In the tetrad formulation of the theory, the antisymmetric part of the tetrad fluctuation transforms as $\delta h_{[a b]}=-\lambda_{a b}+\partial_{[a} \xi_{b]}$, where $\lambda_{a b}$ is the local Lorentz parameter. Maintaining the gauge choice $h_{[a b]}=0$ fixes $\lambda_{a b}=\partial_{[a} \xi_{b]}$. Since $\delta A_{c a b}=\partial_{c} \lambda_{a b}$, one finds (4.7) and hence the source constraint (4.10).
}

$\Pi$, upon solving for $\Phi$ in terms of the source and substituting back into the action, takes the form

$$
\begin{aligned}
\Pi & =-\frac{1}{2} \sum_{J, \mathcal{P}, k, \ell} b_{k \ell}^{-1}\left(J^{\mathcal{P}}\right) \mathcal{J}^{\dagger} \cdot P_{k \ell}\left(J^{\mathcal{P}}\right) \cdot \mathcal{J} \\
& =-\frac{1}{2} \sum_{J, \mathcal{P}, k, \ell} \frac{1}{\operatorname{det} b\left(J^{\mathcal{P}}\right)} C_{k \ell}\left(J^{\mathcal{P}}\right) \mathcal{J}^{\dagger} \cdot P_{k \ell}\left(J^{\mathcal{P}}\right) \cdot \mathcal{J},
\end{aligned}
$$

where $C_{k \ell}$ is the transpose of the cofactor matrix associated with the matrix $b$, which is assumed to have rank $m$. It is important to stress that in our notation $b_{k \ell}^{-1}$ denotes the matrix element of $b^{-1}$ in the representations $k, \ell$, which need not agree with the element of the matrix $b^{-1}$ in the $k$ th row and $\ell$ th column (unless $a$ is nondegenerate, in which case $b=a)$. Given that $b_{i j}(q)$ is a Hermitian matrix and its momentum dependence is polynomial, the poles at nonvanishing values of $q^{2}$ can only come from $\operatorname{det} b\left(J^{\mathcal{P}}\right)$. We assume that for each given $J^{P}$ there will be $s$ propagating particles, with $s \leq m$. Then, we can write

$$
\operatorname{det} b=C\left(q^{2}+m_{1}^{2}\right) \cdots\left(q^{2}+m_{s}^{2}\right),
$$

where $\left(C, m_{1}^{2}, \ldots, m_{s}^{2}\right)$ are constants. For a physical spectrum, these constants must be real, and to simplify the analysis, we shall further assume that the masses $m_{n}^{2}$, $n=1, \ldots, s$, are nonvanishing and distinct (possibly, one of the masses could be zero). The determinant $\operatorname{det} b$ has a simple zero for $q^{2}=-m_{n}^{2}$, so exactly one eigenvalue of $b$ must have a zero there. This implies that the residue matrix

$$
\lim _{q^{2} \rightarrow-m_{n}^{2}}\left(q^{2}+m_{n}^{2}\right) b^{-1}
$$

has exactly one nonvanishing eigenvalue.

Before proceeding to the implication of this for ghostfreedom criteria, we need to first note that the spin projectors in (4.11) contain powers of $1 / q^{2}$ that do not contribute to the physical propagators. These spurious poles at zero momentum, which we shall sometimes refer to as kinematical singularities, cancel out in the full saturated propagator. These poles arise from the product of constants, or $1 /\left(q^{2}+m^{2}\right)$, with the longitudinal parts of the spin projection operators. In the latter case, the simple procedure of partial fractions gives rise to terms in which the spin projection operator are evaluated on the mass shell, plus terms with powers of $1 / q^{2}$. For example,

$$
\frac{1 / q^{2}}{q^{2}+m^{2}}=\frac{1 /\left(-m^{2}\right)}{q^{2}+m^{2}}+\frac{1 / m^{2}}{q^{2}},
$$

and similarly for expressions of the form $1 /\left(\left(q^{2}\right)^{n}\left(q^{2}+m^{2}\right)\right)$. The first term on the rhs has the same pole at $q^{2}=-m^{2}$, but in its coefficient, the momentum squared is now evaluated at the pole. The second term 
gives another spurious pole at zero. In the end, all the spurious poles cancel out, and we are left with a combination of the spin projectors evaluated on the mass shell or constants sandwiched between sources that obey source constraints.

With the issue of kinematical singularities out of the way, we can now state the conditions for the absence of ghosts and tachyons. The tachyon-freedom condition is very simple, namely,

$$
\text { tachyon free } \Rightarrow m_{n}^{2}>0, \quad n=1, \ldots, s .
$$

To examine the ghost-freedom condition, it is convenient to diagonalize the matrix $b^{-1}$. Denoting its eigenvalues by $\lambda^{I}$, and the corresponding eigenvectors by $V^{(I)}$, we have

$$
\Pi=-\frac{1}{2} \sum_{J, \mathcal{P}, k}\left(\sum_{I} \lambda^{I}\left(J^{\mathcal{P}}\right)\left|\hat{\mathcal{J}}_{k}^{(I)}\left(J^{\mathcal{P}}\right)\right|^{2}\right),
$$

where

$$
\hat{\mathcal{J}}_{k}^{(I)}\left(J^{\mathcal{P}}\right)=\sum_{\ell} V_{\ell}^{(I)} P_{k \ell}\left(J^{\mathcal{P}}\right) \cdot \mathcal{J}
$$

Ghost freedom requires that for each value of $k$ the residue of the sum in (4.16) must be negative. As already remarked, precisely one eigenvalue has nonzero residue at a given pole. Thus, noting also that the modulus of the sourcesquared term evaluated at $q^{2}=-m_{n}^{2}$ is finite, we can express the ghost-freedom condition as ${ }^{10}$

ghost free $\Rightarrow \operatorname{trRes}\left(-\left.b^{-1}\right|_{q^{2}=-m_{n}^{2}}\right)>0, \quad n=1, \ldots, s$.

Going back to the formula (4.11), or (4.16), in any $\mathcal{J}^{\mathcal{P}}$ sector involving the matrix $b^{-1}$ with rank greater than 1 , there will clearly be mixing of sources that survive the source constraints. Given that all the kinematical singularities have canceled, the result for the saturated propagator in such $\mathcal{J}^{\mathcal{P}}$ sectors can be written in such a way that the standard form of the spin $J_{P}$ propagators arises in terms of a suitable combination of these sources. This phenomenon will be clearly shown in the multiparameter models analyzed below; see (5.9) and (6.34).

Given any MAG with specific couplings $c_{1} \ldots c_{16}$, $a_{0}, a_{1} \ldots a_{11}$, one can use these conditions on the coefficient matrices given in Appendix B. 1 and determine the spectrum of the theory. However, the 28-parameter class of all MAGs is too broad for a general analysis, so in the following, we discuss two important subclasses: MAGs with either $Q=0$ or $T=0$.

\footnotetext{
${ }^{10}$ The sign depends on the signature of the metric. It may be useful to recall that in our signature, for a massive scalar field, $b=-\left(q^{2}+m^{2}\right)$.
}

\section{THEORIES WITH METRIC CONNECTION}

\section{A. General case}

In metric theories, the following identities hold:

$Q_{\lambda \mu \nu}=0, \quad F_{\mu \nu(\rho \sigma)}=0, \quad F_{\mu \nu}^{(14)}=-F_{\mu \nu}^{(13)}, \quad F_{\mu \nu}=0$.

Using these properties, the most general action up to and including curvature and torsion squared terms is a tenparameter action given by

$$
\begin{aligned}
S(g, A)= & -\frac{1}{2} \int d^{d} x \sqrt{|g|}\left[-a_{0} F+F^{\mu \nu \rho \sigma}\left(g_{1} F_{\mu \nu \rho \sigma}\right.\right. \\
& \left.+g_{3} F_{\rho \sigma \mu \nu}+g_{4} F_{\mu \rho \nu \sigma}\right)+F^{(13) \mu \nu}\left(g_{7} F_{\mu \nu}^{(13)}\right. \\
& \left.+g_{8} F_{\nu \mu}^{(13)}\right)+g_{16} F^{2}+T^{\mu \rho \nu}\left(b_{1} T_{\mu \rho \nu}\right. \\
& \left.\left.+b_{2} T_{\mu \nu \rho}\right)+b_{3} T^{\mu} T_{\mu}\right] .
\end{aligned}
$$

Note that the metricity condition $Q=0$ is a kinematic constraint that changes the nature of the theory: the action (5.2) is not obtained from the general MAG action (2.4) simply by specializing the values of the couplings. Nevertheless, it is useful to write it in the same form and to preserve the numbering of the invariants. To distinguish the two cases, we changed the name of the couplings from $c_{i}$ to $g_{i}$ and from $a_{i}$ to $b_{i}$. Notwithstanding the fact that the action (5.2) is not a special case of (2.4), it is possible to linearize it by making use of the results already computed for the general action (2.4) as follows. Let us first consider the $F^{2}$ terms. In the action (5.2), and in accordance with (5.1), making the substitutions

$F_{a b c d} \rightarrow \frac{1}{2}\left(F_{a b c d}-F_{a b d c}\right), \quad F_{a b}^{(13)} \rightarrow \frac{1}{2}\left(F_{a b}^{(13)}-F_{a b}^{(14)}\right)$

and comparing the result with the general action (2.4), we obtain the relations

$$
\begin{aligned}
c_{1} & =\frac{1}{2} g_{1}, \quad c_{2}=-\frac{1}{2} g_{1}, \quad c_{3}=g_{3}, \quad c_{4}=\frac{1}{4} g_{4}, \\
c_{5} & =-\frac{1}{2} g_{4}, \quad c_{6}=\frac{1}{4} g_{4}, \quad c_{7}=\frac{1}{4} g_{7}, \quad c_{8}=\frac{1}{4} g_{8}, \\
c_{9} & =\frac{1}{4} g_{7}, \quad c_{10}=\frac{1}{4} g_{8}, \quad c_{11}=-\frac{1}{2} g_{7}, \quad c_{12}=-\frac{1}{2} g_{8}, \\
c_{13} & =c_{14}=c_{15}=0, \quad c_{16}=g_{16} .
\end{aligned}
$$

Next, let us consider the substitution required for the parameters $a_{i}$ in terms of $b_{i}$. This is more subtle due to the fact that, expanding around $A_{c a b}=0$, the variation of the metricity condition implies that the fluctuation fields are related by 


$$
\partial_{c} h_{a b}=A_{c a b}+A_{c b a}
$$

where we recall that $A$ denotes also the fluctuation. Thus, inserting in the linearized action the decomposition $A_{c a b}=A_{c[a b]}+A_{c(a b)}$, the symmetric part of $A$ gives terms proportional to $h$ that can be compared to those that, in a general MAG, are produced by $Q$. This gives the relations

$$
\begin{aligned}
& a_{1}=b_{1}, \quad a_{2}=b_{2}, \quad a_{3}=b_{3}, \\
& a_{4}=-a_{5}=\frac{1}{2} b_{1}+\frac{1}{4} b_{2}, \quad a_{6}=a_{7}=\frac{1}{4} b_{3}, \\
& a_{8}=-\frac{1}{2} b_{3}, \quad a_{9}=2 b_{1}+b_{2}, \quad a_{10}=-a_{11}=-b_{3} .
\end{aligned}
$$

In summary, the coefficient matrices of the metric theory are obtained from those of the general MAG by inserting the values for the couplings $c_{i}, a_{i}$ in terms of $g_{i}, b_{i}$ as given in (5.4) and (5.6) and deleting all the rows and columns that pertain to representations carried by symmetric 3-tensors. The remaining representations, and the count of degrees of freedom that they carry, are given in Table IV. The coefficient matrices of metric MAG in $d=4$ are given explicitly in Appendix B. 2.

\section{B. Neville's model}

To test of our formulas and procedures, we reconsider here, as an example, the Neville model [46], which is the same as model ii in Ref. [29]. It corresponds to choosing the couplings $\quad g_{1}=g_{3}=-g_{4} / 4 \equiv g, \quad g_{7}=g_{8}=g_{16}=0$, and $b_{1}=b_{2}=b_{3}=0$.

In the sectors $1^{-}$and $0^{+}$, to fix diffeomorphism invariance, we choose the nondegenerate $b$ matrices to be the upper left $2 \times 2$ submatrices of the general $a$ matrices given in Appendix B. 2, namely, $b_{i j}^{-1}\left(1^{-}\right)$with $i, j=3,6$ and $b_{i j}^{-1}\left(0^{+}\right)$with $i, j=3,5$. The inverses of these coefficient matrices are then given by

TABLE IV. Count of fields of metric MAG: list of irreps of given spin contained in $A$, in $h$, their total number, and number of fields they carry in $d=4$.

\begin{tabular}{lcccc}
\hline \hline$J^{P}$ & $A$ & $h$ & No. of irreps & No. of fields \\
\hline $3^{-}$ & $\cdots$ & $\cdots$ & 0 & 0 \\
$2^{+}$ & 3 & 4 & 2 & 10 \\
$2^{-}$ & 2 & $\cdots$ & 1 & 5 \\
$1^{+}$ & 2,3 & $\cdots$ & 2 & 6 \\
$1^{-}$ & 3,6 & 7 & 3 & 9 \\
$0^{+}$ & 3 & 5,6 & 3 & 3 \\
$0^{-}$ & 1 & $\cdots$ & 1 & 1 \\
Total & & & & 34 \\
\hline \hline
\end{tabular}

$$
\begin{aligned}
& b^{-1}\left(2^{+}\right)=\frac{1}{a_{0}}\left(\begin{array}{cc}
0 & \frac{2 i \sqrt{2}}{|q|} \\
-\frac{2 i \sqrt{2}}{|q|} & -\frac{4}{q^{2}}
\end{array}\right), \quad b^{-1}\left(2^{-}\right)=\frac{2}{a_{0}}, \\
& b^{-1}\left(1^{+}\right)=\frac{1}{a_{0}}\left(\begin{array}{cc}
2 & 0 \\
0 & -1
\end{array}\right), \quad b^{-1}\left(1^{-}\right)=\frac{1}{a_{0}}\left(\begin{array}{cc}
0 & -\sqrt{2} \\
-\sqrt{2} & 1
\end{array}\right), \\
& b^{-1}\left(0^{+}\right)=\frac{1}{a_{0}}\left(\begin{array}{cc}
0 & -\frac{i \sqrt{2}}{|q|} \\
\frac{i \sqrt{2}}{|q|} & \frac{2}{q^{2}}
\end{array}\right), \quad b^{-1}\left(0^{-}\right)=-\frac{1}{a_{0}+6 g q^{2}} .
\end{aligned}
$$

The analysis of Sec. 4.4 shows that this theory contains a massless graviton and a massless pseudoscalar state, with mass $m^{2}=a_{0} /(6 g)$. The absence of tachyons and ghosts requires $a_{0}>0$ and $g>0$. The saturated propagator is

$$
\begin{aligned}
\Pi= & -\frac{1}{2} \int d^{4} q\left\{\mathcal { J } \left(\sum_{i, j=3,4} b_{i j}^{-1}\left(2^{+}\right) P_{i j}\left(2^{+}\right)\right.\right. \\
& \left.+\sum_{i, j=3,5} b_{i j}^{-1}\left(0^{+}\right) P_{i j}\left(0^{+}\right)\right) \mathcal{J}+\tau\left[b^{-1}\left(2^{-}\right) P_{22}\left(2^{-}\right)\right. \\
& +\sum_{i, j=2,3} b_{i j}^{-1}\left(1^{+}\right) P_{i j}\left(1^{+}\right)+\sum_{i, j=3,6} b_{i j}^{-1}\left(1^{-}\right) P_{i j}\left(1^{-}\right) \\
& \left.\left.+b^{-1}\left(0^{-}\right) P\left(0^{-}\right)\right] \tau\right\}
\end{aligned}
$$

As discussed in Sec. 4.4, and using the source constraint (4.10), it can be rewritten in a more explicit form, in which the spin projection operators are put on shell,

$$
\begin{aligned}
\Pi= & -\frac{1}{2 a_{0}} \int d q\left\{\tau \cdot\left(-\frac{m^{2}}{q^{2}+m^{2}} P\left(0^{-}, m^{2}\right)+2 P_{22}\left(2^{-}, \eta\right)\right) \cdot \tau\right. \\
& \left.-\frac{4}{q^{2}} S \cdot\left(P_{44}\left(2^{+}, \eta\right)-\frac{1}{2} P_{55}\left(0^{+}, \eta\right)\right) \cdot S\right\}
\end{aligned}
$$

where $S_{a b}=\sigma_{a b}+i q^{c} \tau_{a c b} \sigma_{a b}$, and following Ref. [29], we have used

$$
\begin{gathered}
\left.P\left(J^{\mathcal{P}}, m^{2}\right) \equiv P\left(J^{\mathcal{P}}, q\right)\right|_{q^{2}=-m^{2}} . \\
\left.P\left(J^{\mathcal{P}}, \eta\right) \equiv P\left(J^{\mathcal{P}}, q\right)\right|_{\partial \rightarrow 0} .
\end{gathered}
$$

The last term is the standard graviton propagator

$$
\int d q S^{a b}(-q) \frac{2}{a_{0} q^{2}}\left(\eta_{a c} \eta_{b d}-\frac{1}{2} \eta_{a b} \eta_{c d}\right) S^{c d}(q),
$$

while for the spin $0^{-}$, we have 


$$
\tau \cdot P\left(0^{-}, m^{2}\right) \cdot \tau=\tau^{[c a b]}\left(\eta_{c d}+\frac{q_{c} q_{d}}{m^{2}}\right)\left(\eta_{a e}+\frac{q_{a} q_{e}}{m^{2}}\right)\left(\eta_{b f}+\frac{q_{b} q_{f}}{m^{2}}\right) \tau^{[d e f]}
$$

The spin $1^{+}$and $1^{-}$contributions actually vanish.

\section{TORSION-FREE THEORIES}

\section{A. General case}

In torsion-free theories, the following identities hold:

$$
T_{\mu}^{\rho_{\nu}}=0, \quad F_{[\mu \nu}{ }^{\rho}{ }_{\sigma]}=0, \quad F_{\mu \nu}=-2 F_{[\mu \nu]}^{(13)} .
$$

These reduce the number of independent invariants. One finds that the terms in (2.4) with parameters $c_{5}, c_{6}, c_{13}, c_{14}, c_{15}, a_{1}$, $a_{2}, a_{3}, a_{9}, a_{10}, a_{11}$ become redundant. Thus, we parametrize the most general torsion-free MAG action as

$$
\begin{aligned}
S(g, A)= & -\frac{1}{2} \int d^{d} x \sqrt{|g|}\left[-a_{0} F+F^{\mu \nu \rho \sigma}\left(h_{1} F_{\mu \nu \rho \sigma}+h_{2} F_{\mu \nu \sigma \rho}+h_{3} F_{\rho \sigma \mu \nu}+h_{4} F_{\mu \rho \nu \sigma}\right)\right. \\
& +F^{(13) \mu \nu}\left(h_{7} F_{\mu \nu}^{(13)}+h_{8} F_{\nu \mu}^{(13)}\right)+F^{(14) \mu \nu}\left(h_{9} F_{\mu \nu}^{(14)}+h_{10} F_{\nu \mu}^{(14)}\right)+F^{(14) \mu \nu}\left(h_{11} F_{\mu \nu}^{(13)}+h_{12} F_{\nu \mu}^{(13)}\right)+h_{16} F^{2} \\
& \left.+Q^{\rho \mu \nu}\left(a_{4} Q_{\rho \mu \nu}+a_{5} Q_{\nu \mu \rho}\right)+a_{6} Q^{\mu} Q_{\mu}+a_{7} \tilde{Q}^{\mu} \tilde{Q}_{\mu}+a_{8} Q^{\mu} \tilde{Q}_{\mu}\right] .
\end{aligned}
$$

Once again, we note that $T=0$ is a kinematic constraint, so the theories we now consider are not equivalent to just setting to zero the parameters listed above. For this reason, the remaining parameters $c_{i}$ have been renamed $h_{i}$.

In the torsion-free case, the field $A_{\lambda \mu \nu}$ is symmetric in $\lambda$, $\nu$. In four dimensions, this reduces the number of degrees of freedom of $A$ from 64 to 40. The corresponding spin representations are listed in the second column of Table V. To obtain the coefficient matrices, we use the "primed" spin projectors defined in the end of Sec. III. B, which are better suited to decompose a tensor symmetric in the first and last indices. All the primed spin projectors in the columns $h a$ and $t a$ in Table II give zero when acting on a torsion-free connection. Thus, the coefficient matrices for this case are smaller: their dimensions are given by the fourth column of Table IV. A diffeomorphism (2.13) preserves the symmetry of $A_{\lambda \mu \nu}$, and diffeomorphism symmetry reduces by 1 the rank of the coefficient matrices for spins $1^{-}$and $0^{+}$. The coefficient matrices for the torsion-free theory in four dimensions are given in Appendix B. 3.

\section{B. Torsion-free theories with projective symmetry}

Let us now examine the possible additional symmetries in this case. We find that, while the symmetry (2.20) is still too restrictive, in the sense that it requires all $c$ coefficients to vanish, we can achieve projective symmetry, which is now a symmetric combination of (2.17) and (2.23):

$$
\delta_{4} A_{\mu}{ }^{\rho}{ }_{\nu}=2 \lambda_{(\mu} \delta_{\nu)}^{\rho}, \quad \delta g_{\mu \nu}=0
$$

It follows that

$$
\begin{aligned}
\delta_{4} F_{\mu \nu \rho \sigma} & =2 g_{\rho \sigma} \nabla_{[\mu} \lambda_{\nu]}-2 g_{\rho[\mu} \nabla_{\nu]} \lambda_{\sigma}, \\
\delta_{4} Q_{\rho \mu \nu} & =2 \lambda_{\rho} g_{\mu \nu}+2 g_{\rho(\mu} \lambda_{\nu} .
\end{aligned}
$$

TABLE V. Count of fields of torsion-free MAG: list of irreps of given spin contained in $A$, in $h$, their total number, and number of fields they carry in $d=4$.

\begin{tabular}{lcccc}
\hline \hline$J^{P}$ & $A$ & $h$ & No. of irreps & No. of fields \\
\hline $3^{-}$ & 1 & $\ldots$ & 1 & 7 \\
$2^{+}$ & 1,2 & 4 & 3 & 15 \\
$2^{-}$ & 1 & $\ldots$ & 1 & 5 \\
$1^{+}$ & 1 & $\ldots$ & 1 & 3 \\
$1^{-}$ & $1,2,4,5$ & 7 & 5 & 15 \\
$0^{+}$ & $1,2,4$ & 5,6 & 5 & 5 \\
$0^{-}$ & $\cdots$ & $\cdots$ & 0 & 0 \\
Total & & & & 50 \\
\hline \hline
\end{tabular}


Invariance of the action is found to require that

$$
\begin{aligned}
h_{1} & =\frac{1}{4}\left[-2 h_{7}+2 d h_{8}+(d-1) h_{11}+(d+2) h_{12}+2(1-d) h_{16}\right], \\
h_{3} & =\left[2 h_{2}+\frac{d}{2}\left(h_{11}+h_{12}\right)+(1-d) h_{16}\right], \\
h_{4} & =\frac{1}{2}\left[-4 h_{2}+2(2-d) h_{7}+2(1-2 d) h_{8}-2 d h_{11}-(2 d+3) h_{12}+4(d-1) h_{16}\right], \\
h_{9} & =\frac{1}{6}\left[2(d-2) h_{7}+2(2 d-1) h_{8}+(d+1) h_{11}+(2 d+5) h_{12}+6(1-d) h_{16}\right], \\
h_{10} & =\frac{1}{6}\left[-2(d-2) h_{7}-2(2 d-1) h_{8}-(d-2) h_{11}-2(d+1) h_{12}\right], \\
a_{4} & =\frac{1}{16}\left[5(1-d) a_{0}-24(d+1) a_{6}+4(d+3) a_{7}-2(d+7) a_{8}\right], \\
a_{5} & =\frac{1}{8}\left[3(d-1) a_{0}+8(d+1) a_{6}-4(d+3) a_{7}+2(1-d) a_{8}\right],
\end{aligned}
$$

where we have used (6.1) and the formula

$$
\delta_{4} \int d^{d} x \sqrt{-g} F=\int d^{d} x \sqrt{-g}(1-d)\left(\frac{1}{2} Q_{\mu}-\tilde{Q}_{\mu}\right) \lambda^{\mu}
$$

with a total derivative term discarded. The part of the action proportional to $h_{2}$ vanishes due to the identity

$$
F^{\mu \nu \rho \sigma}\left(F_{\mu \nu \sigma \rho}+2 F_{\rho \sigma \mu \nu}-2 F_{\mu \rho \nu \sigma}\right)=0
$$

which follows from repeated use of the second equation in (6.1). Therefore, the action depends on nine parameters, namely, $\left(a_{0}, a_{6}, a_{7}, a_{8}\right)$ and $\left(h_{7}, h_{8}, h_{11}, h_{12}, h_{16}\right)$, and it takes the form

$$
\begin{aligned}
S(g, A)= & -\frac{1}{2} \int d^{d} x \sqrt{|g|}\left\{-a_{0} F+F^{\mu \nu \rho \sigma}\left(\gamma_{1} F_{\mu \nu \rho \sigma}+\gamma_{2} F_{\mu \rho \nu \sigma}+\gamma_{3} F_{\rho \sigma \mu \nu}\right)+h_{16} F^{2}\right. \\
& +F^{(13) \mu \nu}\left(h_{7} F_{\mu \nu}^{(13)}+h_{8} F_{\nu \mu}^{(13)}+h_{11} F_{\mu \nu}^{(14)}+h_{12} F_{\nu \mu}^{(14)}\right)+F^{(14) \mu \nu}\left(\gamma_{4} F_{\mu \nu}^{(14)}+\gamma_{5} F_{\nu \mu}^{(14)}\right) \\
& \left.+Q^{\rho \mu \nu}\left(\gamma_{6} Q_{\rho \mu \nu}+\gamma_{7} Q_{\nu \mu \rho}\right)+a_{6} Q_{\mu} Q^{\mu}+a_{7} \tilde{Q}^{\mu} \tilde{Q}_{\mu}+a_{8} Q^{\mu} \tilde{Q}_{\mu}\right),
\end{aligned}
$$

where the parameters $\left(\gamma_{1}, \ldots, \gamma_{7}\right)$ are defined in terms of the nine parameters of the action as

$$
\begin{aligned}
& \gamma_{1}=-\frac{1}{2} h_{7}+\frac{d}{2} h_{8}+\frac{d-1}{4} h_{11}+\frac{d+2}{4} h_{12}+\frac{1-d}{2} h_{16}, \\
& \gamma_{2}=(2-d) h_{7}+(1-2 d) h_{8}-d h_{11}-\frac{2 d+3}{2} h_{12}+2(d-1) h_{16}, \\
& \gamma_{3}=\frac{d}{2}\left(h_{11}+h_{12}\right)+(1-d) h_{16}, \\
& \gamma_{4}=\frac{d-2}{3} h_{7}+\frac{2 d-1}{3} h_{8}+\frac{d+1}{6} h_{11}+\frac{2 d+5}{6} h_{12}+(1-d) h_{16}, \\
& \gamma_{5}=\frac{2-d}{3} h_{7}+\frac{1-2 d}{3} h_{8}+\frac{2-d}{6} h_{11}-\frac{d+1}{3} h_{12}, \\
& \gamma_{6}=\frac{5(1-d)}{16} a_{0}-\frac{3(d+1)}{2} a_{6}+\frac{d+3}{4} a_{7}-\frac{d+7}{8} a_{8} \\
& \gamma_{7}=\frac{3(d-1)}{8} a_{0}+(d+1) a_{6}-\frac{d+3}{2} a_{7}+\frac{1-d}{4} a_{8} .
\end{aligned}
$$

In four dimensions, the projective symmetry eliminates four fields, reducing by 1 the ranks of the coefficient matrices $1^{-}$ and $0^{+}$. In fact, one finds that $a\left(1^{-}\right)$has the null eigenvectors 
$(\sqrt{10 / 3} i|q|, \sqrt{2 / 3} i|q|, \sqrt{3 / 2} i|q|, 0,1),(\sqrt{10}, \sqrt{2}, \sqrt{2}, 1,0)$,

while $a\left(0^{+}\right)$has the null eigenvectors

$$
(-(1 / 2) i|q|,(1 / 2 \sqrt{2}) i|q|, 0,0,1),(1,-1 / \sqrt{2}, 1,0,0) .
$$

The ranks of the coefficient matrices for the representations $3^{-}, 2^{+}, 2^{-}, 1^{+}, 1^{-}, 0^{+}$are $1,3,1,1,3,3$, respectively.

Invariance of the source term implies that the sources must obey the constraints:

$$
\tau_{\nu \mu}^{\nu}=0, \tau_{\mu \nu}^{\nu}=0
$$

Next, we examine the spectrum of this nine-parameter model.

\section{New ghost- and tachyon-free theories}

To further simplify matters, we shall restrict our attention to choices of parameters such that:

(i) The spin-3 field does not propagate.

(ii) In the spin- $2^{+}$sector, only the massless graviton propagates.

Condition $\mathrm{i}$ is achieved by setting to zero the coefficient of $-q^{2}$ in (B14):

$$
h_{16}=\frac{1}{6}\left(6 h_{7}+6 h_{8}+5 h_{11}+5 h_{12}\right) \text {. }
$$

To impose ii, we consider the rank-3 matrix $b_{i j}\left(2^{+}\right)=$ $a_{i j}\left(2^{+}\right)$with $i, j=1,2,4$. Demanding that the determinant of this matrix contains no powers of $-q^{2}$ higher than 1 leads to

$$
h_{12}=-h_{11}, \quad h_{8}=-h_{7} .
$$

With these conditions, the class of actions that we consider is of the form

$$
\begin{aligned}
S(g, A)= & -\frac{1}{2} \int d^{n} x \sqrt{|g|}\left\{-a_{0} F-\frac{1}{4}\left(10 h_{7}+3 h_{11}\right) F^{\mu \nu \rho \sigma}\left(F_{\mu \nu \rho \sigma}-2 F_{\mu \rho \nu \sigma}\right)\right. \\
& +2 F_{[\mu \nu]}^{(13)}\left(h_{7} F^{(13) \mu \nu}+h_{11} F^{(14) \mu \nu}\right)-\frac{2}{3}\left(5 h_{7}+4 h_{11}\right) F_{[\mu \nu]}^{(14)} F^{(14) \mu \nu} \\
& +\frac{1}{48}\left(12 a_{0}+A-16 B\right) Q^{\rho \mu \nu} Q_{\rho \mu \nu}-\frac{1}{24}\left(12 a_{0}-A-8 B\right) Q^{\rho \mu \nu} Q_{\nu \mu \rho} \\
& -\frac{1}{288}\left(72 a_{0}+A-32 B+49 C\right) Q_{\mu} Q^{\mu}-\frac{1}{72}(A-8 B+25 C) \tilde{Q}_{\mu} \tilde{Q}^{\mu} \\
& \left.+\frac{1}{72}\left(36 a_{0}-A-16 B+35 C\right) Q_{\mu} \tilde{Q}^{\mu}\right\},
\end{aligned}
$$

where we introduced the following convenient new combinations of parameters:

$$
\begin{aligned}
& A=7 a_{0}-40 a_{6}-28 a_{7}-34 a_{8}, \\
& B=4 a_{0}+20 a_{6}-7 a_{7}+2 a_{8}, \\
& C=a_{0}+8 a_{6}-4 a_{7}+2 a_{8} .
\end{aligned}
$$

Let us now discuss the dynamical content of this theory. We have already ruled out the propagation of a spin-3 state, for which

$$
a\left(3^{-}\right)=-\frac{A}{4} .
$$

In the spin- $2^{+}$sector, we have

$$
\operatorname{det} b\left(2^{+}\right)=\frac{1}{32} a_{0} A B q^{2} .
$$

As is well known, the propagation of a massless spin- $2^{+}$ state requires an admixture of a spin- $0^{+}$state. Having imposed (6.13) and (6.14), and fixing the diffeomorphism and projective gauges by choosing the nondegenerate coefficient submatrix to be $b_{i j}\left(0^{+}\right)$with $i, j=3,4,5$, we get

$$
\operatorname{det} b\left(0^{+}\right)=-\frac{1}{16} a_{0} A C q^{2} .
$$

Thus, the existence of a massless graviton requires that $A, B$, $C$, and $a_{0}$ are all nonvanishing. In particular, this implies that the coefficient matrix for the spin-3 sector is not zero.

As we shall now see, having imposed (6.13) and (6.14), we find that all the coefficient matrices have maximum rank submatrices whose determinants are at most first order in $q^{2}$. This means that in any given sector at most one state propagates. Indeed, denoting $b\left(2^{-}\right)=a\left(2^{-}\right)_{11}, b\left(1^{+}\right)=$ $a\left(1^{+}\right)_{11}$, and taking the nondegenerate submatrix $b_{i j}\left(1^{-}\right)$ with $i, j=2,4,5$, we find 


$$
\begin{gathered}
b\left(2^{-}\right)=\frac{1}{4}\left[2 B+\left(30 h_{7}+9 h_{11}\right) q^{2}\right] . \\
b\left(1^{+}\right)=\frac{1}{6}\left[3 B+\left(40 h_{7}+17 h_{11}\right) q^{2}\right], \\
\operatorname{det} b\left(1^{-}\right)=-\frac{5 A}{288} \Delta, \quad \text { where } \Delta=6 B C+(16 B+25 C)\left(2 h_{7}+h_{11}\right) q^{2} .
\end{gathered}
$$

Note that, since $A, B, C$ are nonvanishing, there is no room for accidental symmetries. From these equations, we read off the masses of the modes $2^{-}, 1^{+}$, and $1^{-}$:

$$
m_{2}^{2}=\frac{2 B}{30 h_{7}+9 h_{11}}, \quad m_{+}^{2}=\frac{3 B}{40 h_{7}+17 h_{11}}, \quad m_{-}^{2}=\frac{6 B C}{(16 B+25 C)\left(2 h_{7}+h_{11}\right)},
$$

We can now list the matrices $b_{i j}^{-1}\left(J^{\mathcal{P}}\right)$ :

$$
\begin{aligned}
& b^{-1}\left(3^{-}\right)=-\frac{4}{A}, \\
& b^{-1}\left(2^{+}\right)=\frac{1}{a_{0} q^{2}}\left(\begin{array}{ccc}
-\frac{1}{3} q^{2} & \frac{2 \sqrt{2}}{3} q^{2} & -\frac{2}{\sqrt{3}} i|q| \\
\frac{2 \sqrt{2}}{3} q^{2} & -\frac{8}{3} q^{2} & 4 \sqrt{\frac{2}{3}} i|q| \\
\frac{2}{\sqrt{3}} i|q| & -4 \sqrt{\frac{2}{3}} i|q| & -4
\end{array}\right)+\left(\begin{array}{ccc}
-\frac{4}{A} & 0 & 0 \\
0 & \frac{2}{B} & 0 \\
0 & 0 & 0
\end{array}\right), \\
& b^{-1}\left(0^{+}\right)=\frac{1}{a_{0} q^{2}}\left(\begin{array}{ccc}
\frac{3}{4} q^{2} & \frac{1}{2 \sqrt{2}} q^{2} & -\sqrt{3 / 2} i|q| \\
\frac{1}{2 \sqrt{2}} q^{2} & \frac{1}{6} q^{2} & -\frac{1}{\sqrt{3}} i|q| \\
\sqrt{3 / 2} i|q| & \frac{1}{\sqrt{3}} i|q| & 2
\end{array}\right)+\left(\begin{array}{ccc}
\frac{1}{C}-\frac{1}{A} & \frac{2 \sqrt{2}}{A} & 0 \\
\frac{2 \sqrt{2}}{A} & -\frac{8}{A} & 0 \\
0 & 0 & 0
\end{array}\right) \text {. } \\
& b^{-1}\left(2^{-}\right)=\frac{4}{2 B+\left(30 h_{7}+9 h_{11}\right) q^{2}}, \\
& b^{-1}\left(1^{+}\right)=\frac{6}{3 B+\left(40 h_{7}+17 h_{11}\right) q^{2}}, \\
& b^{-1}\left(1^{-}\right)=\frac{1}{\Delta}\left(\begin{array}{ccc}
4(B+C)+\frac{2}{3}\left(2 h_{7}+h_{11}\right) q^{2} & 0 & \frac{\sqrt{2}}{3}\left(6 B-12 C+13\left(2 h_{7}+h_{11}\right) q^{2}\right) \\
0 & 0 & 0 \\
\frac{\sqrt{2}}{3}\left(6 B-12 C+13\left(2 h_{7}+h_{11}\right) q^{2}\right) & 0 & 2 B+8 C+\frac{169}{3}\left(2 h_{7}+h_{11}\right) q^{2}
\end{array}\right) \\
& -\frac{1}{15 A}\left(\begin{array}{ccc}
2 & 12 & \sqrt{2} \\
12 & 72 & 6 \sqrt{2} \\
\sqrt{2} & 6 \sqrt{2} & 1
\end{array}\right)
\end{aligned}
$$

We can now state the ghost- and tachyon-free conditions. The tachyon-free conditions amount to the positivity of the masses (6.23), which are equivalent to

$$
\begin{gathered}
\left(10 h_{7}+3 h_{11}\right) B>0, \quad\left(40 h_{7}+17 h_{11}\right) B>0, \\
B C(16 B+25 C)\left(2 h_{7}+h_{11}\right)>0 .
\end{gathered}
$$


Applying the formula (4.18), one finds that the ghost-free conditions for the spin- $2^{+},-2^{-},-1^{+}$, and $-1^{-}$sectors, are given by

$$
\begin{aligned}
& a_{0}>0, \quad 10 h_{7}+3 h_{11}<0, \\
& 40 h_{7}+17 h_{11}<0, \quad\left(2 h_{7}+h_{11}\right)<0 .
\end{aligned}
$$

All these conditions together are equivalent to $a_{0}>0, \quad B<0, C(16 B+25 C)>0, \quad$ and

$h_{11}<-2 h_{7} \quad$ for $h_{7} \leq 0, \quad$ or $\quad h_{11}<-\frac{10}{3} h_{7}$ for

$h_{7}>0$.

Finally, the saturated propagator is

$$
\begin{aligned}
\Pi= & -\frac{1}{2} \int d^{4} q\left\{\mathcal{J}\left(\sum_{i, j=1,2,4} b_{i j}^{-1}\left(2^{+}\right) P_{i j}\left(2^{+}\right)+\sum_{i, j=3,4,5} b_{i j}^{-1}\left(0^{+}\right) P_{i j}\left(0^{+}\right)\right) \mathcal{J}\right. \\
& \left.+\tau\left[b^{-1}\left(3^{-}\right) P\left(3^{-}\right)+b^{-1}\left(2^{-}\right) P_{11}\left(2^{-}\right)+b^{-1}\left(1^{+}\right) P_{11}\left(1^{+}\right)+\sum_{i, j=2,4,5} b^{-1}\left(1^{-}\right)_{i j} P_{i j}\left(1^{-}\right)\right] \tau\right\} .
\end{aligned}
$$

We can make this expression more understandable by explicitly displaying the denominators of each propagator and evaluating the contractions of the spin projectors with the sources,

$$
\begin{aligned}
\Pi= & -\frac{1}{2} \int d^{4} q\left\{-\frac{4}{A} \tau \cdot P\left(3^{-}, \eta\right) \cdot \tau-\frac{1}{a_{0} q^{2}} S_{a b}\left(P_{44}^{a b c d}\left(2^{+}, \eta\right)-\frac{1}{2} P_{55}^{a b c d}\left(0^{+}, \eta\right)\right) S_{c d}\right. \\
& +\frac{16}{30 h_{7}+9 h_{11}} \frac{1}{q^{2}+m_{2}^{2}} \tau \cdot P_{11}\left(2^{-}, m_{2}^{2}\right) \cdot \tau \\
& -\frac{1}{2 B} \frac{1}{q^{2}+m_{+}^{2}} \operatorname{div}_{1} \tau_{[a b]}\left(\eta^{a c}+\frac{q^{a} q^{c}}{m_{+}^{2}}\right)\left(\eta^{b d}+\frac{q^{b} q^{d}}{m_{+}^{2}}\right) \operatorname{div}_{1} \tau_{[c d]} \\
& -\frac{1}{6 B^{2}(16 B+25 C)^{2}\left(2 h_{7}+h_{11}\right)} \frac{1}{q^{2}+m_{-}^{2}} Z_{a}\left(\eta^{a b}+\frac{q^{a} q^{b}}{m_{-}^{2}}\right) Z_{b} \\
& \left.+\frac{1}{45}\left(\frac{1}{A}-\frac{5}{16 B+25 C}\right) \operatorname{tr}_{13} \tau_{a} \operatorname{tr}_{13} \tau^{a}\right\},
\end{aligned}
$$

where we defined

$$
\begin{gathered}
S_{a b}=2 i \operatorname{div}_{1} \tau_{a b}-i \operatorname{div}_{2} \tau_{a b}-2 \sigma_{a b}, \\
Z_{a}=(16 B+25 C)\left(2 h_{7}+h_{11}\right)\left(\operatorname{div}_{12} \tau_{a}-\operatorname{div}_{13} \tau_{a}\right)-2 B(4 B+5 C) \operatorname{tr}_{13} \tau_{a},
\end{gathered}
$$

and

$$
\begin{aligned}
& \operatorname{div}_{1} \tau_{a b}=q^{c} \tau_{c a b}, \quad \operatorname{div}_{2} \tau_{a b}=q^{c} \tau_{a c b}, \\
& \operatorname{div}_{12} \tau_{a}=q^{b} q^{c} \tau_{c b a}, \quad \operatorname{div}_{13} \tau_{a}=q^{b} q^{c} \tau_{c a c}, \quad \operatorname{tr}_{13} \tau_{a}=\tau_{c a}{ }^{c} .
\end{aligned}
$$

This manifestly shows the spin- $2^{+},-1^{+}$, and $-1^{-}$degrees of freedom being sourced by suitable combinations of sources. In particular, we note that the spin- $2^{+}$and $-1^{-}$degrees of freedom have propagators of the standard form. The propagator for the spin $1^{+}$seems less familiar, but it is simply that of a massive two-form potential, described by the Lagrangian

$$
\mathcal{L}=-\frac{1}{12} H_{\mu \nu \rho} H^{\mu \nu \rho}-\frac{1}{2} m^{2} B_{\mu \nu} B^{\mu \nu}
$$

where $H_{\mu \nu \rho}=3 \partial_{[\mu} B_{\nu \rho]}$. We also note that, unlike the case of spin $2^{+}$, the spin-2- propagator cannot be written solely in terms of second-rank tensor sources, as it necessarily requires the presence of the third-rank sources. 


\section{CONCLUSIONS}

In this paper, we have set up the machinery that is necessary to analyze the spectrum of a general MAG theory. In particular, we have constructed the spin projectors for a general three-index tensor, and we have used them to rewrite the wave operator for the most general, 28parameter MAG. Not surprisingly, this case turns out to be too complicated to determine its spectrum, but it is possible to do so in special subclasses of theories. We have considered here theories that have either vanishing nonmetricity, recovering previously known results, and theories with vanishing torsion. In the latter case, the theory depends on 17 parameters; imposing projective invariance reduces this to ten parameters, and imposing that there be no propagating spin- $3^{-}$and no massive propagating spin$2^{+}$fields further reduces this number to 6 . The absence of ghosts and tachyons results in the inequalities (6.32) on these six parameters. Even within the torsion-free subtheory, relaxing the conditions of Sec. VI.3 will lead to a much more complicated system.

With hindsight, the absence of ghosts and tachyons in these models is related the fact that, when converting to the $R, \phi$ variables in the manner of Eq. (1.1), they do not contain any terms quadratic in curvature. For the same reason, these models are also nonrenormalizable. This is entirely analogous to the situation also pointed out in Ref. [29] for the nine-parameter metric quadratic theories with torsion. Similarly, we expect that allowing a propagating massive spin- $2^{+}$mode will probably make the theory renormalizable but not unitary.

It is important to stress that the metric and torsion-free cases are kinematically distinct from the original general MAG and that the ghost- and tachyon-free models we have found are not special cases of the general MAG, but only of the kinematically restricted models. In fact, some classes of ghost- and tachyon-free Poincaré gauge theories that are different from our six parameter ghost- and tachyon-free model have been found in Ref. [34]. We leave it for future work to study special subclasses of the general MAG.

Also of some interest would be the study of models with propagating spin $3^{-}$. It is known that the free massless spin3 theory can be embedded in linearized MAG [49]; however, the underlying linearized gauge symmetry does not extend to the full theory. It would be interesting to explore whether MAG can describe a massive spin-3 field coupled to gravity. We hope to return to these questions in the future.

\section{ACKNOWLEDGMENTS}

E. S. thanks Mitya Ponomarev for useful discussions. R.P. would like to thank the Mitchell Institute for
Fundamental Physics and Astronomy, and E. S. thanks International School for Advanced Studies (SISSA) for hospitality. This work is supported in part by NSF Grants No. PHY-1521099 and No. PHY-1803875 and by Mitchell Institute for Fundamental Physics and Astronomy.

Note added.-Recently, we were informed that the spin projectors for the general theory have also been worked out in Ref. [50] and that they agree with ours.

\section{APPENDIX A: SPIN PROJECTORS}

In the torsion-free case, the spin projectors have also been given in Ref. [5].

\section{1. $P\left(J^{-}\right)$projectors, $J=0,1,2,3$}

Let us introduce the notation

$\Pi_{i}:=\left(\Pi^{(t s)}, \Pi^{(h s)}, \Pi^{(h a)}, \Pi^{(t a)}, \Pi^{(s)}, \Pi^{a}\right), \quad i=1,2, \ldots, 6$,

where we recall that $\Pi^{(t s)}, \ldots, \Pi^{a}$ are defined in (3.5), (3.6), and (3.7).

The negative parity projectors are given by

$$
\begin{aligned}
& \left(3^{-}\right)=\Pi_{1}(T T T) \Pi_{1}-P\left(1^{-}\right)_{11}, \\
P\left(2^{-}\right)_{i-1, j-1} & =\Pi_{i} A_{i j} \Pi_{j}-P\left(1^{-}\right)_{i j}, \quad i, j=2,3, \\
P\left(1^{-}\right)_{i j} & =\Pi_{i} B_{i j} \Pi_{j}, \quad i, j=1,2,3, \\
P\left(1^{-}\right)_{i, 3+j} & =\Pi_{i} B_{i, 3+j} \Pi_{j}, \quad i, j=1,2,3, \\
P\left(1^{-}\right)_{3+i, 3+j} & =\Pi_{i} B_{i j} \Pi_{j}, i, j=1,2,3, \\
P\left(1^{-}\right)_{i 7} & =\Pi_{i} B_{i 7} \Pi_{5}, P\left(1^{-}\right)_{3+i, 7}=\Pi_{i} B_{3+i, 7} \Pi_{5}, i=1,2,3, \\
P\left(1^{-}\right)_{77} & =\Pi_{5}(T L+L T) \Pi_{5}, \\
P\left(0^{-}\right) & =\Pi_{4}(T T T) \Pi_{4},
\end{aligned}
$$

where it is understood that there is no summation over the indices displayed, and

$$
A\left(2^{-}\right)_{i j}:=\left(\begin{array}{cc}
1 & \sqrt{2} \\
\sqrt{2} & 1
\end{array}\right) T T T,
$$




$$
\begin{aligned}
& B\left(1^{-}\right)_{i j}:=\left(\begin{array}{ccc}
\frac{3}{d+1} & \frac{3 \sqrt{2}}{\sqrt{(d-2)(d+1)}} & \frac{\sqrt{6}}{\sqrt{(d-2)(d+1)}} \\
\frac{3 \sqrt{2}}{\sqrt{(d-2)(d+1)}} & \frac{6}{d-2} & \frac{2 \sqrt{3}}{d-2} \\
\frac{\sqrt{6}}{\sqrt{(d-2)(d+1)}} & \frac{2 \sqrt{3}}{d-2} & \frac{2}{d-2}
\end{array}\right) T_{12} T^{12} T \\
& B\left(1^{-}\right)_{i, 3+j}:=\left(\begin{array}{ccc}
\frac{3}{\sqrt{d+1}} & \frac{3 \sqrt{2}}{\sqrt{d+1}} & \frac{\sqrt{6}}{\sqrt{d+1}} \\
\frac{3 \sqrt{2}}{\sqrt{d+1}} & \frac{6}{\sqrt{d-2}} & \frac{2 \sqrt{3}}{\sqrt{d-2}} \\
\frac{\sqrt{6}}{\sqrt{d+1}} & \frac{2 \sqrt{3}}{\sqrt{d-2}} & \frac{2}{\sqrt{d-2}}
\end{array}\right) L_{12} T^{12} T \\
& B\left(1^{-}\right)_{3+i, 3+j}:=\left(\begin{array}{ccc}
L L T+L T L+T L L & 3 \sqrt{2} L L T & \sqrt{6} L L T \\
3 \sqrt{2} L L T & L L T+L T L+T L L & 2 \sqrt{3} L L T \\
\sqrt{6} L L T & 2 \sqrt{3} L L T & L L T+L T L+T L L
\end{array}\right) \text {, } \\
& {\left[B\left(1^{-}\right)_{i 7}\right]_{c a b}{ }^{e f}:=\left(\begin{array}{c}
\frac{\sqrt{6}}{\sqrt{d+1}} T_{23} \hat{q}^{\prime} T \\
-\frac{\sqrt{3}}{\sqrt{d-2}} T_{23} \hat{q}^{\prime} T \\
\frac{2}{\sqrt{d-2}} T_{31} \hat{q}^{\prime} T
\end{array}\right), \quad\left[B\left(1^{-}\right)_{3+i, 7}\right]_{c a b}{ }^{e f}:=\left(\begin{array}{c}
\sqrt{6} L_{23} \hat{q}^{\prime} T \\
-\sqrt{3} L_{23} \hat{q}^{\prime} T \\
2 L_{31} \hat{q}^{\prime} T
\end{array}\right) .}
\end{aligned}
$$

Note that the transposition raises and lowers the vector indices on $T$ and $L$ such that, for example, $T_{c}{ }^{d}$ and $T_{c a}$ get mapped to $T_{d}^{c}$ and $T^{c a}$, respectively. Therefore, we have $\left(B^{T}\right)_{d e f}{ }^{c a b}=T_{d e} L^{c a} T_{f}^{b}$.

\section{2. $P\left(J^{+}\right)$projectors, $J=0,1,2$}

The positive parity projectors are given by

$$
\begin{aligned}
& P\left(2^{+}\right)_{i j}=\Pi_{i} C_{i j} \Pi_{j}-P\left(0^{+}\right)_{i j}, \quad P\left(2^{+}\right)=\Pi C_{i 4} \Pi_{5}, \quad P\left(2^{+}\right)_{44}=\Pi_{5}(T T) \Pi_{5}-P\left(0^{+}\right)_{55}, \\
& P\left(1^{+}\right)_{i-1, j-1}=\Pi_{i} D_{i j} \Pi_{j}, \quad i, j=2,3,4, \\
& P\left(0^{+}\right)_{i j}=\Pi_{i} E_{i j} \Pi_{j}, \quad P\left(0^{+}\right)_{i 4}=\Pi_{i} E_{i 4} \Pi_{1}, \quad P\left(0^{+}\right)_{44}=\Pi_{1}(L L L) \Pi_{1}, \\
& P\left(0^{+}\right)_{i r}=\Pi_{i} E_{i r} \Pi_{5}, \quad P\left(0^{+}\right)_{4 r}=\Pi_{1} E_{4 r} \Pi_{5}, \quad P\left(0^{+}\right)_{r s}=\Pi_{5} E_{r s} \Pi_{5}, \\
& C\left(2^{+}\right)_{i j}:=\left(\begin{array}{ccc}
T T L+T L T+L T T & \frac{3}{2 \sqrt{2}} L T T & \sqrt{6} T L T \\
\frac{3}{2 \sqrt{2}} L T T & \frac{3}{2} L T T & \Pi_{5}(T T) L \\
\sqrt{6} T L T & \Pi_{5}(T T) L & T T L+T L T-\frac{1}{2} L T T
\end{array}\right), \\
& C\left(2^{+}\right)_{i 4}:=\left(\begin{array}{c}
\sqrt{3} \hat{q}_{c} T_{a}^{e} T_{b}^{f} \\
\sqrt{\frac{3}{2}} T_{a}{ }^{e} T_{b}^{f} \\
\frac{1}{\sqrt{2}} T_{c}{ }^{e} \hat{q}_{a} T_{b}^{f}
\end{array}\right),
\end{aligned}
$$




$$
\begin{aligned}
& D\left(1^{+}\right)_{i j}:=\left(\begin{array}{ccc}
T T L+T L T-\frac{1}{2} L T T & 2 \sqrt{3} \Pi_{6}(T T) L & \sqrt{6} T T L \\
2 \sqrt{3} \Pi_{6}(T T) L & \frac{3}{2} L T T & -\frac{3}{\sqrt{2}} L T T \\
\sqrt{6} T T L & -\frac{3}{\sqrt{2}} L T T & T T L+T L T+L T T
\end{array}\right), \\
& E\left(0^{+}\right)_{i j}:=\frac{1}{d-1}\left(\begin{array}{ccc}
3 L T_{23} T^{23} & \frac{3}{\sqrt{2}} L T_{23} T^{23} & \sqrt{6} T_{31} T_{31} L \\
\frac{3}{\sqrt{2}} L T_{23} T^{23} & \frac{3}{2} L T_{23} T^{23} & 2 \sqrt{3} T_{12} T^{12} L \\
\sqrt{6} T_{31} T_{31} L & 2 \sqrt{3} T_{12} T^{12} L & 2 T_{12} T^{12} L
\end{array}\right), \\
& E\left(0^{+}\right)_{i 4}:=\frac{1}{\sqrt{d-1}}\left(\begin{array}{c}
\sqrt{3} L T_{23} T^{23} \\
\sqrt{\frac{3}{2}} L T_{23} T^{23} \\
-\sqrt{2} T_{12} T^{12} L
\end{array}\right) \text {, } \\
& E\left(0^{+}\right)_{i r}:=\left(\begin{array}{cc}
\frac{\sqrt{3}}{d-1} \hat{q} T_{23} T^{23} & \frac{\sqrt{3}}{\sqrt{d-1}} \hat{q} T_{23} L^{23} \\
\frac{\sqrt{3}}{2(d-1)} \hat{q} T_{23} T^{23} & \frac{\sqrt{3}}{\sqrt{d-1}} \hat{q} T_{23} L^{23} \\
\frac{\sqrt{3}}{d-1} \hat{q} T_{31} T^{12} & \frac{\sqrt{2}}{\sqrt{d-1}} \hat{q} T_{31} L^{12}
\end{array}\right), \quad E\left(0^{+}\right)_{4 r}:=\left(\begin{array}{c}
\frac{\sqrt{2}}{\sqrt{d-1}} L_{12} \hat{q} T^{23} \\
L_{12} \hat{q} L^{23}
\end{array}\right), \\
& E\left(0^{+}\right)_{r s}:=\left(\begin{array}{cc}
\frac{1}{d-1} T_{23} T^{23} & \frac{1}{\sqrt{d-1}} T_{23} L^{23} \\
\frac{1}{\sqrt{d-1}} L_{23} T^{23} & L_{23} L^{23}
\end{array}\right), \quad i=1,2,3, \quad r, s=5,6 .
\end{aligned}
$$

\section{APPENDIX B: COEFFICIENT MATRICES}

\section{General MAG}

Here, we provide the coefficient matrices $a\left(J^{P}\right)$ arising in the expansion of the wave operator in the general 28-parameter model, in terms of the spin projection operators. For a weak check, we observe that all coefficient matrices vanish identically for the combination (2.6).

$$
\begin{gathered}
a\left(3^{-}\right)=\left(2 c_{1}+2 c_{2}+c_{4}+c_{5}+c_{6}\right)\left(-q^{2}\right)-a_{0}-4 a_{4}-4 a_{5} \\
a\left(2^{-}\right)_{11}=2\left(c_{1}+c_{2}-c_{4}-c_{5}-c_{6}\right)\left(-q^{2}\right)+\frac{1}{2}\left(a_{0}-6 a_{1}-3 a_{2}-8 a_{4}+4 a_{5}-6 a_{9}\right) \\
a\left(2^{-}\right)_{12}=\frac{\sqrt{3}}{2}\left[\left(c_{4}-c_{6}\right)\left(-q^{2}\right)-2 a_{1}-a_{2}-a_{9}\right] \\
a\left(2^{-}\right)_{22}=\left(2\left(c_{1}-c_{2}\right)+\frac{1}{2}\left(c_{4}-c_{5}+c_{6}\right)\right)\left(-q^{2}\right)+\frac{1}{2} a_{0}-a_{1}-\frac{1}{2} a_{2} \\
a\left(2^{+}\right)_{11}=\left(\frac{2}{3}\left(2 c_{1}+2 c_{2}+c_{4}+c_{5}+c_{6}\right)+\frac{1}{3}\left(c_{7}+c_{8}+c_{9}+c_{10}+c_{11}+c_{12}\right)\right)\left(-q^{2}\right)-a_{0}-4 a_{4}-4 a_{5} \\
a\left(2^{+}\right)_{12}=-\frac{1}{3 \sqrt{2}}\left[2\left(2 c_{1}+2 c_{2}+c_{4}+c_{5}+c_{6}\right)+c_{7}+c_{8}+c_{9}+c_{10}+c_{11}+c_{12}\right]\left(-q^{2}\right) \\
a\left(2^{+}\right)_{13}=\frac{1}{\sqrt{6}}\left(c_{7}+c_{8}-c_{9}-c_{10}\right)\left(-q^{2}\right) \\
a\left(2^{+}\right)_{14}=\frac{1}{2 \sqrt{3}}\left(a_{0}+4 a_{4}+4 a_{5}\right) i|q|
\end{gathered}
$$




$$
\begin{aligned}
a\left(2^{+}\right)_{22}= & \left(\frac{1}{3}\left(2 c_{1}+2 c_{2}+c_{4}+c_{5}+c_{6}\right)+\frac{1}{6}\left(c_{7}+c_{8}+c_{9}+c_{10}+c_{11}+c_{12}\right)\right)\left(-q^{2}\right) \\
& +\frac{1}{2} a_{0}-3 a_{1}-\frac{3}{2} a_{2}-4 a_{4}+2 a_{5}-3 a_{9} \\
a\left(2^{+}\right)_{23}= & \left.\frac{1}{2 \sqrt{3}}\left[\left(-c_{7}-c_{8}+c_{9}+c_{10}\right)\left(-q^{2}\right)-6 a_{1}-3 a_{2}-3 a_{9}\right)\right] \\
a\left(2^{+}\right)_{24}= & \frac{1}{2 \sqrt{6}}\left(-a_{0}+8 a_{4}-4 a_{5}+3 a_{9}\right) i|q| \\
a\left(2^{+}\right)_{33}= & \left(\left(2 c_{1}-2 c_{2}+2 c_{3}+c_{4}-c_{5}+c_{6}\right)+\frac{1}{2}\left(c_{7}+c_{8}+c_{9}+c_{10}-c_{11}-c_{12}\right)\right)\left(-q^{2}\right)+\frac{1}{2} a_{0}-a_{1}-\frac{1}{2} a_{2} \\
a\left(2^{+}\right)_{34}= & \frac{1}{2 \sqrt{2}}\left(a_{0}+a_{9}\right) i|q| \\
a\left(2^{+}\right)_{44}= & -a_{4} q^{2}
\end{aligned}
$$

$$
\begin{aligned}
& a\left(1^{-}\right)_{11}=\left(2 c_{1}+2 c_{2}+c_{4}+c_{5}+c_{6}+\frac{5}{3}\left(c_{7}+c_{9}+c_{11}+2 c_{13}-c_{14}-c_{15}\right)\right)\left(-q^{2}\right) \\
& +\frac{2}{3} a_{0}-4 a_{4}-4 a_{5}-\frac{20}{3}\left(a_{6}+a_{7}+a_{8}\right) \\
& a\left(1^{-}\right)_{12}=\frac{\sqrt{5}}{6}\left[\left(2 c_{7}+2 c_{9}+2 c_{11}-8 c_{13}+c_{14}+c_{15}\right)\left(-q^{2}\right)+2 a_{0}+16 a_{6}-8 a_{7}+4 a_{8}-6 a_{10}-6 a_{11}\right] \\
& a\left(1^{-}\right)_{13}=\frac{\sqrt{5}}{2 \sqrt{3}}\left[\left(2 c_{7}-2 c_{9}-c_{14}+c_{15}\right)\left(-q^{2}\right)-2 a_{10}-2 a_{11}\right] \\
& a\left(1^{-}\right)_{14}=\frac{\sqrt{5}}{3}\left[\left(-c_{8}-c_{10}-c_{12}+2 c_{13}-c_{14}-c_{15}\right)\left(-q^{2}\right)+a_{0}-4 a_{6}-4 a_{7}-4 a_{8}\right] \\
& a\left(1^{-}\right)_{15}=\frac{\sqrt{5}}{\sqrt{18}}\left[2\left(c_{8}+c_{10}+c_{12}-2 c_{13}+c_{14}+c_{15}\right)\left(-q^{2}\right)+a_{0}+8 a_{6}-4 a_{7}+2 a_{8}-3 a_{10}-3 a_{11}\right] \\
& a\left(1^{-}\right)_{16}=-\frac{\sqrt{5}}{\sqrt{6}}\left(a_{10}+a_{11}\right) \\
& a\left(1^{-}\right)_{17}=\sqrt{\frac{5}{24}}\left(-a_{0}+4 a_{7}+2 a_{8}\right) i|q| \\
& a\left(1^{-}\right)_{22}=\frac{1}{6}\left(12 c_{1}+12 c_{2}-3 c_{4}-3 c_{5}-3 c_{6}+2 c_{7}+2 c_{9}+2 c_{11}+16 c_{13}+4 c_{14}+4 c_{15}\right)\left(-q^{2}\right) \\
& +\frac{5}{6} a_{0}-3 a_{1}-\frac{3}{2} a_{2}-3 a_{3}-4 a_{4}+2 a_{5}-\frac{16}{3} a_{6}-\frac{4}{3} a_{7}+\frac{8}{3} a_{8}-3 a_{9}+4 a_{10}-2 a_{11} \\
& a\left(1^{-}\right)_{23}=\frac{1}{2 \sqrt{3}}\left[\left(3 c_{4}-3 c_{6}+2 c_{7}-2 c_{9}+2 c_{14}-2 c_{15}\right)\left(-q^{2}\right)-6 a_{1}-3 a_{2}-6 a_{3}-3 a_{9}+4 a_{10}-2 a_{11}\right] \\
& a\left(1^{-}\right)_{24}=\frac{1}{6}\left[\left(-2 c_{8}-2 c_{10}-2 c_{12}-8 c_{13}+c_{14}+c_{15}\right)\left(-q^{2}\right)+2 a_{0}+16 a_{6}-8 a_{7}+4 a_{8}-6 a_{10}-6 a_{11}\right] \\
& a\left(1^{-}\right)_{25}=\frac{1}{3 \sqrt{2}}\left[\left(2 c_{8}+2 c_{10}+2 c_{12}+8 c_{13}-c_{14}-c_{15}\right)\left(-q^{2}\right)\right. \\
& \left.+a_{0}-9 a_{3}-16 a_{6}-4 a_{7}+8 a_{8}+12 a_{10}-6 a_{11}\right] \\
& a\left(1^{-}\right)_{26}=\frac{1}{\sqrt{6}}\left(-3 a_{3}+2 a_{10}-a_{11}\right)
\end{aligned}
$$




$$
\begin{aligned}
& a\left(1^{-}\right)_{27}=\frac{1}{2 \sqrt{6}}\left(-a_{0}+4 a_{7}-4 a_{8}+3 a_{11}\right) i|q| \\
& a\left(1^{-}\right)_{33}=\frac{1}{2}\left[\left(4 c_{1}-4 c_{2}+c_{4}-c_{5}+c_{6}+2 c_{7}+2 c_{9}-2 c_{11}\right)\left(-q^{2}\right)-a_{0}-2 a_{1}-a_{2}-2 a_{3}\right] \\
& a\left(1^{-}\right)_{34}=\frac{1}{2 \sqrt{3}}\left[\left(-2 c_{8}+2 c_{10}-c_{14}+c_{15}\right)\left(-q^{2}\right)-2 a_{10}-2 a_{11}\right] \\
& a\left(1^{-}\right)_{35}=\frac{1}{\sqrt{6}}\left[\left(2 c_{8}-2 c_{10}+c_{14}-c_{15}\right)\left(-q^{2}\right)-3 a_{3}+2 a_{10}-a_{11}\right] \\
& a\left(1^{-}\right)_{36}=-\frac{1}{\sqrt{2}}\left(a_{0}+a_{3}\right) \\
& a\left(1^{-}\right)_{37}=\frac{1}{2 \sqrt{2}}\left(-a_{0}+a_{11}\right) i|q| \\
& a\left(1^{-}\right)_{44}=\frac{1}{3}\left[\left(2 c_{1}+2 c_{2}+c_{4}+c_{5}+c_{6}+c_{7}+c_{9}+c_{11}+2 c_{13}-c_{14}-c_{15}\right)\left(-q^{2}\right)\right. \\
& \left.-2 a_{0}-12 a_{4}-12 a_{5}-4 a_{6}-4 a_{7}-4 a_{8}\right] \\
& a\left(1^{-}\right)_{45}=\frac{1}{3 \sqrt{2}}\left[-2\left(2 c_{1}+2 c_{2}+c_{4}+c_{5}+c_{6}+c_{7}+c_{9}+c_{11}+2 c_{13}-c_{14}-c_{15}\right)\left(-q^{2}\right)\right. \\
& \left.+a_{0}+8 a_{6}-4 a_{7}+2 a_{8}-3 a_{10}-3 a_{11}\right] \\
& a\left(1^{-}\right)_{46}=-\frac{1}{\sqrt{6}}\left(a_{10}+a_{11}\right) \\
& a\left(1^{-}\right)_{47}=\frac{1}{2 \sqrt{6}}\left(a_{0}+8 a_{4}+8 a_{5}+4 a_{7}+2 a_{8}\right) i|q| \\
& a\left(1^{-}\right)_{55}=\frac{2}{3}\left(2 c_{1}+2 c_{2}+c_{4}+c_{5}+c_{6}+c_{7}+c_{9}+c_{11}+2 c_{13}-c_{14}-c_{15}\right)\left(-q^{2}\right) \\
& +\frac{2}{3} a_{0}-3 a_{1}-\frac{3}{2} a_{2}-\frac{3}{2} a_{3}-4 a_{4}+2 a_{5}-\frac{8}{3} a_{6}-\frac{2}{3} a_{7}+\frac{4}{3} a_{8}-3 a_{9}+2 a_{10}-a_{11} \\
& a\left(1^{-}\right)_{56}=\frac{1}{2 \sqrt{3}}\left(-6 a_{1}-3 a_{2}-3 a_{3}-3 a_{9}+2 a_{10}-a_{11}\right) \\
& a\left(1^{-}\right)_{57}=\frac{1}{4 \sqrt{3}}\left[-2 a_{0}+8 a_{4}-4 a_{5}+4 a_{7}-4 a_{8}+3 a_{9}+3 a_{11}\right] i|q| \\
& a\left(1^{-}\right)_{66}=-\frac{1}{2}\left(2 a_{1}+a_{2}+a_{3}\right) \\
& a\left(1^{-}\right)_{67}=\frac{1}{4}\left(a_{9}+a_{11}\right) i|q| \\
& a\left(1^{-}\right)_{77}=\frac{1}{2}\left(2 a_{4}+a_{5}+a_{7}\right)\left(-q^{2}\right) \\
& a\left(1^{+}\right)_{11}=\frac{1}{2}\left[\left(4 c_{1}+4 c_{2}+c_{7}-c_{8}+c_{9}-c_{10}+c_{11}-c_{12}\right)\left(-q^{2}\right)\right. \\
& \left.+a_{0}-6 a_{1}-3 a_{2}-8 a_{4}+4 a_{5}-6 a_{9}\right] \\
& a\left(1^{+}\right)_{12}=-\frac{1}{2 \sqrt{3}}\left[\left(2 c_{4}-2 c_{6}+c_{7}-c_{8}-c_{9}+c_{10}\right)\left(-q^{2}\right)-6 a_{1}-3 a_{2}-3 a_{9}\right] \\
& a\left(1^{+}\right)_{13}=\frac{1}{\sqrt{6}}\left(-2 c_{4}+2 c_{6}-c_{7}+c_{8}+c_{9}-c_{10}\right)\left(-q^{2}\right) \\
& a\left(1^{+}\right)_{22}=\frac{1}{6}\left(4 c_{1}-4 c_{2}-4 c_{3}+c_{7}-c_{8}+c_{9}-c_{10}-c_{11}+c_{12}\right)\left(-q^{2}\right)+\frac{1}{2} a_{0}-a_{1}-\frac{1}{2} a_{2}
\end{aligned}
$$




$$
\begin{aligned}
& a\left(1^{+}\right)_{23}=\frac{1}{3 \sqrt{2}}\left(4 c_{1}-4 c_{2}-4 c_{3}+c_{7}-c_{8}+c_{9}-c_{10}-c_{11}+c_{12}\right)\left(-q^{2}\right) \\
& a\left(1^{+}\right)_{33}=\frac{1}{3}\left(4 c_{1}-4 c_{2}-4 c_{3}+c_{7}-c_{8}+c_{9}-c_{10}-c_{11}+c_{12}\right)\left(-q^{2}\right)-a_{0}-4 a_{1}+4 a_{2} \\
& a\left(0^{+}\right)_{11}=\frac{2}{3}\left(2 c_{1}+2 c_{2}+c_{4}+c_{5}+c_{6}+2 c_{7}+2 c_{8}+2 c_{9}+2 c_{10}+2 c_{11}+2 c_{12}\right)\left(-q^{2}\right) \\
& -4\left(a_{4}+a_{5}+a_{6}+a_{7}+a_{8}\right) \\
& a\left(0^{+}\right)_{12}=\frac{1}{3 \sqrt{2}}\left[-2\left(2 c_{1}+2 c_{2}+c_{4}+c_{5}+c_{6}+2 c_{7}+2 c_{8}+2 c_{9}+2 c_{10}+2 c_{11}+2 c_{12}\right)\left(-q^{2}\right)\right. \\
& \left.-3 a_{0}-24 a_{6}+12 a_{7}-6 a_{8}+9 a_{10}+9 a_{11}\right] \\
& a\left(0^{+}\right)_{13}=\frac{1}{\sqrt{6}}\left[2\left(-c_{7}-c_{8}+c_{9}+c_{10}\right)\left(-q^{2}\right)+3\left(a_{10}+a_{11}\right)\right] \\
& a\left(0^{+}\right)_{14}=a_{0}-4\left(a_{6}+a_{7}+a_{8}\right) \\
& a\left(0^{+}\right)_{15}=\frac{1}{2 \sqrt{3}}\left(a_{0}+4 a_{4}+4 a_{5}+12 a_{6}+6 a_{8}\right) i|q| \\
& a\left(0^{+}\right)_{16}=\left(-\frac{1}{2} a_{0}+2 a_{6}+2 a_{7}+2 a_{8}\right) i|q| \\
& a\left(0^{+}\right)_{22}=\frac{1}{3}\left(2 c_{1}+2 c_{2}+c_{4}+c_{5}+c_{6}+2 c_{7}+2 c_{8}+2 c_{9}+2 c_{10}+2 c_{11}+2 c_{12}\right)\left(-q^{2}\right) \\
& +a_{0}-3 a_{1}-\frac{3}{2} a_{2}-\frac{9}{2} a_{3}-4 a_{4}+2 a_{5}-8 a_{6}-2 a_{7}+4 a_{8}-3 a_{9}+6 a_{10}-3 a_{11} \\
& a\left(0^{+}\right)_{23}=\frac{1}{2 \sqrt{3}}\left[2\left(c_{7}+c_{8}-c_{9}-c_{10}\right)\left(-q^{2}\right)-6 a_{1}-3\left(a_{2}+3 a_{3}+a_{9}\right)+6 a_{10}-3 a_{11}\right] \\
& a\left(0^{+}\right)_{24}=\frac{1}{\sqrt{2}}\left[-a_{0}-8 a_{6}+4 a_{7}-2 a_{8}+3 a_{10}+3 a_{11}\right] \\
& a\left(0^{+}\right)_{25}=\frac{1}{2 \sqrt{6}}\left(-a_{0}+8 a_{4}-4 a_{5}+24 a_{6}-6 a_{8}+3 a_{9}-9 a_{10}\right) i|q| \\
& a\left(0^{+}\right)_{26}=\frac{1}{2 \sqrt{2}}\left(a_{0}+8 a_{6}-4 a_{7}+2 a_{8}-3 a_{10}-3 a_{11}\right) i|q| \\
& a\left(0^{+}\right)_{33}=\left(2 c_{1}-2 c_{2}+2 c_{3}+c_{4}-c_{5}+c_{6}+2 c_{7}+2 c_{8}+2 c_{9}+2 c_{10}-2 c_{11}-2 c_{12}+6 c_{16}\right)\left(-q^{2}\right) \\
& -a_{0}-a_{1}-\frac{1}{2} a_{2}-\frac{3}{2} a_{3} \\
& a\left(0^{+}\right)_{34}=\sqrt{\frac{3}{2}}\left(a_{10}+a_{11}\right) \quad a\left(0^{+}\right)_{35}=\frac{1}{2 \sqrt{2}}\left(-2 a_{0}+a_{9}-3 a_{10}\right) i|q| \\
& a\left(0^{+}\right)_{36}=-\frac{\sqrt{3}}{2 \sqrt{2}}\left(a_{10}+a_{11}\right) i|q| \\
& a\left(0^{+}\right)_{44}=-4\left(a_{4}+a_{5}+a_{6}+a_{7}+a_{8}\right) \\
& a\left(0^{+}\right)_{45}=\sqrt{3}\left(2 a_{6}+a_{8}\right) i|q| \quad a\left(0^{+}\right)_{46}=2\left(a_{4}+a_{5}+a_{6}+a_{7}+a_{8}\right) i|q| \\
& a\left(0^{+}\right)_{55}=\left(a_{4}+3 a_{6}\right)\left(-q^{2}\right) \quad a\left(0^{+}\right)_{56}=\frac{\sqrt{3}}{2}\left(2 a_{6}+a_{8}\right)\left(-q^{2}\right) \\
& a\left(0^{+}\right)_{66}=\left(a_{4}+a_{5}+a_{6}+a_{7}+a_{8}\right)\left(-q^{2}\right) \\
& a\left(0^{-}\right)=\left(2 c_{1}-2 c_{2}-c_{4}+c_{5}-c_{6}\right)\left(-q^{2}\right)-a_{0}-4 a_{1}+4 a_{2} .
\end{aligned}
$$




\section{Metric MAG}

Using (5.4) and (5.6) in the coefficient matrices of Appendix B.1, and deleting the rows and columns that pertain to symmetric 3-tensors, we recover the coefficient matrices of the metric theory, as computed in Ref. [29] (see also Ref. [51]) with two differences. First, one has to keep in mind that the graviton field $h_{a b}$ used here is equal to one-half of the graviton field $\varphi_{a b}$ used in those references. This gives a factor 2 in the mixed $A-h$ coefficients and 4 in the $h-h$ coefficients. Second, the projectors $P_{i j}\left(1^{+}\right)$with $i, j=2,3$ span the same space as $P_{i j}\left(1^{+}\right)$with $i, j=1,2$ in those references but differ by a linear transformation [the old projectors do not respect the $G L(4)$ decomposition]. This is of no consequence for the physical results.

$$
\begin{gathered}
a\left(2^{-}\right)=\frac{1}{2}\left(\left(4 g_{1}+g_{4}\right)\left(-q^{2}\right)+a_{0}-2 b_{1}-b_{2}\right) \\
a\left(2^{+}\right)_{33}=\frac{1}{2}\left(\left(4 g_{1}+4 g_{3}+2 g_{4}+g_{7}+g_{8}\right)\left(-q^{2}\right)+a_{0}-2 b_{1}-b_{2}\right) \\
a\left(2^{+}\right)_{34}=\frac{i|q|}{2 \sqrt{2}}\left(a 0+2 b_{1}+b_{2}\right), \quad a\left(2^{+}\right)_{44}=\frac{1}{4}\left(2 b_{1}+b_{2}\right)\left(-q^{2}\right) \\
a\left(1^{+}\right)_{22}=\frac{1}{6}\left(4 g_{1}-4 g_{3}+g_{7}-g_{8}\right)\left(-q^{2}\right)+\frac{1}{2} a_{0}-2 b_{1}+b_{2} \\
a\left(1^{+}\right)_{23}=\frac{4 g_{1}-4 g_{3}+g_{7}-g_{8}}{3 \sqrt{2}}\left(-q^{2}\right) \\
a\left(1^{+}\right)_{33}=\frac{1}{3}\left(4 g_{1}-4 g_{3}+g_{7}-g_{8}\right)\left(-q^{2}\right)-a_{0}+8 b_{1}-8 b_{2} \\
a\left(1^{-}\right)_{33}=\frac{1}{2}\left(\left(4 g_{1}+g_{4}+2 g_{7}\right)\left(-q^{2}\right)-a_{0}-2 b_{1}-b_{2}-2 b_{3}\right) \\
a\left(1^{-}\right)_{36}=-\frac{a_{0}+b_{3}}{\sqrt{2}}, \quad a\left(1^{-}\right)_{37}=\frac{i|q|}{2 \sqrt{2}}\left(-a_{0}+b_{3}\right) \\
a\left(1^{-}\right)_{66}=-\frac{1}{2}\left(2 b_{1}+b_{2}+b_{3}\right), \quad a\left(1^{-}\right)_{67}=\frac{i|q|}{4}\left(2 b_{1}+b_{2}+b_{3}\right) \\
a\left(1^{-}\right)_{77}=\frac{1}{8}\left(2 b_{1}+b_{2}+b_{3}\right)\left(-q^{2}\right) \\
a\left(0^{+}\right)_{55}=\frac{1}{4}\left(2 b_{1}+b_{2}+3 b_{3}\right)\left(-q^{2}\right) \\
a\left(0^{+}\right)_{56}=a\left(0^{+}\right)_{66}=0 \\
a\left(0^{+}\right)_{33}=\left(2 g_{1}+2 g_{3}+g_{4}+2 g_{7}+2 g_{8}+6 g_{16}\right)\left(-q^{2}\right)+\frac{1}{2}\left(-2 a_{0}-2 b_{1}-b_{2}-3 b_{3}\right) \\
=-\frac{i|q|}{2 \sqrt{2}}\left(2 a_{0}-2 b_{1}-b_{2}-3 b_{3}\right) \\
\left.a 0^{-}\right)=\left(2 g_{1}-g_{4}\right)\left(-q^{2}\right)-a_{0}-4 b_{1}+4 b_{2} . \\
a-10
\end{gathered}
$$

\section{Torsion-free MAG}

We give here the coefficient matrices for the most general torsion-free model, as discussed in Sec. VI. A. If one wishes to further impose the projective symmetry discussed in Sec. VI. B, one has to further impose (in four dimensions) the conditions given in Eq. (6.5).

Beware of our notational convention; the indices $i, j$ on the coefficient matrix $a_{i j}\left(J^{\mathcal{P}}\right)$ refer to the representations they carry. Thus, they do not always agree with the usual convention of numbering matrix elements. For example, the representation $2_{3}^{+}$is absent from the symmetric tensor $A_{c a b}$; only the representations 1, 2, 4 are present. Accordingly, the element of $a\left(2^{+}\right)$in the third row and column is labeled $a_{44}\left(2^{+}\right)$. 


$$
\begin{aligned}
& a\left(3^{-}\right)=\left(2 h_{1}+2 h_{2}+h_{4}\right)\left(-q^{2}\right)-a_{0}-4 a_{4}-4 a_{5} \\
& a\left(2^{+}\right)_{11}=\frac{1}{3}\left(4 h_{1}+4 h_{2}+2 h_{4}+h_{7}+h_{8}+h_{9}+h_{10}+h_{11}+h_{12}\right)\left(-q^{2}\right)-a_{0}-4 a_{4}-4 a_{5} \\
& a\left(2^{+}\right)_{12}=\frac{1}{6 \sqrt{2}}\left[4 h_{1}+4 h_{2}+2 h_{4}+4 h_{7}+4 h_{8}-2 h_{9}-2 h_{10}+h_{11}+h_{12}\right]\left(-q^{2}\right) \\
& a\left(2^{+}\right)_{14}=\frac{1}{2 \sqrt{3}}\left(a_{0}+4 a_{4}+4 a_{5}\right) i|q| \\
& a\left(2^{+}\right)_{22}=\frac{1}{6}\left(10 h_{1}-8 h_{2}+9 h_{3}+5 h_{4}+4 h_{7}+4 h_{8}+h_{9}+h_{10}-2 h_{11}-2 h_{12}\right)\left(-q^{2}\right)+\frac{1}{2}\left(a_{0}-2 a_{4}+a_{5}\right) \\
& a\left(2^{+}\right)_{24}=\frac{1}{\sqrt{6}}\left(a_{0}-2 a_{4}+a_{5}\right) i|q|, a\left(2^{+}\right)_{44}=a_{4}\left(-q^{2}\right) \text {. } \\
& a\left(2^{-}\right)_{11}=\left(2 h_{1}-h_{2}-\frac{1}{2} h_{4}\right)\left(-q^{2}\right)+\frac{1}{2} a_{0}-a_{4}+\frac{1}{2} a_{5} \\
& a\left(1^{+}\right)_{11}=\frac{1}{2}\left[\left(2 h_{1}-h_{3}-h_{4}+h_{9}-h_{10}\right)\left(-q^{2}\right)+a_{0}-2 a_{4}+a_{5}\right] \\
& a\left(1^{-}\right)_{11}=\frac{1}{3}\left(6 h_{1}+6 h_{2}+3 h_{4}+5 h_{7}+5 h_{9}+5 h_{11}\right)\left(-q^{2}\right)+\frac{2}{3} a_{0}-4 a_{4}-4 a_{5}-\frac{20}{3}\left(a_{6}+a_{7}+a_{8}\right) \\
& a\left(1^{-}\right)_{12}=-\frac{\sqrt{5}}{6}\left[\left(-2 h_{7}+4 h_{9}+h_{11}\right)\left(-q^{2}\right)+a_{0}+8 a_{6}-4 a_{7}+2 a_{8}\right] \\
& a\left(1^{-}\right)_{14}=\frac{\sqrt{5}}{3}\left[\left(-h_{8}-h_{10}-h_{12}\right)\left(-q^{2}\right)+a_{0}-4 a_{6}-4 a_{7}-4 a_{8}\right] \\
& a\left(1^{-}\right)_{15}=-\frac{\sqrt{5}}{6 \sqrt{2}}\left[2\left(h_{8}+h_{10}+h_{12}\right)\left(-q^{2}\right)+a_{0}+8 a_{6}-4 a_{7}+2 a_{8}\right] \\
& a\left(1^{-}\right)_{17}=\frac{\sqrt{5}}{2 \sqrt{6}}\left(-a_{0}+4 a_{7}+2 a_{8}\right) i|q| \\
& a\left(1^{-}\right)_{22}=\frac{1}{6}\left[\left(12 h_{1}-6 h_{2}-3 c_{4}+2 h_{7}-8 h_{9}+4 h_{11}\right)\left(-q^{2}\right)-a_{0}-6 a_{4}+3 a_{5}-8 a_{6}-2 a_{7}+4 a_{8}\right] \\
& a\left(1^{-}\right)_{24}=-\frac{1}{6}\left[\left(2 h_{8}-4 h_{10}-h_{12}\right)\left(-q^{2}\right)+a_{0}+8 a_{6}-4 a_{7}+2 a_{8}\right] \\
& a\left(1^{-}\right)_{25}=\frac{1}{6 \sqrt{2}}\left[\left(-2 h_{8}+4 h_{10}+h_{12}\right)\left(-q^{2}\right)-4 a_{0}-8 a_{6}-2 a_{7}+4 a_{8}\right] \\
& a\left(1^{-}\right)_{27}=-\frac{1}{2 \sqrt{6}}\left(a_{0}+2 a_{7}-2 a_{8}\right) i|q| \\
& a\left(1^{-}\right)_{44}=\frac{1}{3}\left[\left(2 h_{1}+2 h_{2}+h_{4}+h_{7}+h_{9}+h_{11}\right)\left(-q^{2}\right)-2 a_{0}-12 a_{4}-12 a_{5}-4 a_{6}-4 a_{7}-4 a_{8}\right] \\
& a\left(1^{-}\right)_{45}=\frac{1}{6 \sqrt{2}}\left[2\left(2 h_{1}+2 h_{2}+h_{4}+h_{7}+h_{9}+h_{11}\right)\left(-q^{2}\right)-a_{0}-8 a_{6}+4 a_{7}-2 a_{8}\right] \\
& a\left(1^{-}\right)_{47}=\frac{1}{2 \sqrt{6}}\left(a_{0}+8 a_{4}+8 a_{5}+4 a_{7}+2 a_{8}\right) i|q|
\end{aligned}
$$




$$
\begin{aligned}
& a\left(1^{-}\right)_{55}=\frac{1}{6}\left[\left(2 h_{1}+2 h_{2}+h_{4}+h_{7}+h_{9}+h_{11}\right)\left(-q^{2}\right)+a_{0}-6 a_{4}+3 a_{5}-4 a_{6}-a_{7}+2 a_{8}\right] \\
& a\left(1^{-}\right)_{57}=\frac{1}{4 \sqrt{3}}\left(a_{0}-4 a_{4}+2 a_{5}-2 a_{7}+2 a_{8}\right) i|q| \\
& a\left(1^{-}\right)_{77}=\frac{1}{2}\left(2 a_{4}+a_{5}+a_{7}\right)\left(-q^{2}\right) \\
& a\left(0^{+}\right)_{11}=\frac{2}{3}\left(2 h_{1}+2 h_{2}+h_{4}+2 h_{7}+2 h_{8}+2 h_{9}+2 h_{10}+2 h_{11}+2 h_{12}\right)\left(-q^{2}\right) \\
& -4\left(a_{4}+a_{5}+a_{6}+a_{7}+a_{8}\right) \\
& a\left(0^{+}\right)_{12}=\frac{1}{6 \sqrt{2}}\left[2\left(2 h_{1}+2 h_{2}+h_{4}-h_{7}-h_{8}+5 h_{9}+5 h_{10}+2 h_{11}+2 h_{12}\right)\left(-q^{2}\right)\right. \\
& \left.+3 a_{0}+24 a_{6}-12 a_{7}+6 a_{8}\right] \\
& a\left(0^{+}\right)_{14}=a_{0}-4\left(a_{6}+a_{7}+a_{8}\right) \\
& a\left(0^{+}\right)_{15}=\frac{1}{2 \sqrt{3}}\left(a_{0}+4 a_{4}+4 a_{5}+12 a_{6}+6 a_{8}\right) i|q|, \quad a\left(0^{+}\right)_{16}=\frac{1}{2}\left(-a_{0}+4 a_{6}+4 a_{7}+4 a_{8}\right) i|q| \\
& a\left(0^{+}\right)_{22}=\frac{1}{6}\left(10 h_{1}-8 h_{2}+9 h_{3}+5 h_{4}+7 h_{7}+7 h_{8}+13 h_{9}+13 h_{10}-8 h_{11}-8 h_{12}+27 h_{16}\right)\left(-q^{2}\right) \\
& +\frac{1}{2}\left(-a_{0}-2 a_{4}+a_{5}-4 a_{6}-a_{7}+2 a_{8}\right) \\
& a\left(0^{+}\right)_{24}=\frac{1}{2 \sqrt{2}}\left[a_{0}+8 a_{6}-4 a_{7}+2 a_{8}\right], \quad a\left(0^{+}\right)_{25}=-\frac{1}{4 \sqrt{6}}\left(5 a_{0}+8 a_{4}-4 a_{5}+24 a_{6}-6 a_{8}\right) i|q| \\
& a\left(0^{+}\right)_{26}=-\frac{1}{4 \sqrt{2}}\left(a_{0}+8 a_{6}-4 a_{7}+2 a_{8}\right) i|q| \\
& a\left(0^{+}\right)_{44}=-4\left(a_{4}+a_{5}+a_{6}+a_{7}+a_{8}\right) \quad a\left(0^{+}\right)_{45}=\sqrt{3}\left(2 a_{6}+a_{8}\right) i|q| \\
& a\left(0^{+}\right)_{46}=2\left(a_{4}+a_{5}+a_{6}+a_{7}+a_{8}\right) i|q|, \quad a\left(0^{+}\right)_{55}=\left(a_{4}+3 a_{6}\right)\left(-q^{2}\right) \\
& a\left(0^{+}\right)_{56}=\frac{\sqrt{3}}{2}\left(2 a_{6}+a_{8}\right)\left(-q^{2}\right), \quad a\left(0^{+}\right)_{66}=\left(a_{4}+a_{5}+a_{6}+a_{7}+a_{8}\right)\left(-q^{2}\right) \\
& a\left(0^{-}\right)=0 .
\end{aligned}
$$

[1] F. W. Hehl, J. D. McCrea, E. W. Mielke, and Y. Ne'eman, Metric affine gauge theory of gravity: Field equations, Noether identities, world spinors, and breaking of dilation invariance, Phys. Rep. 258, 1 (1995).

[2] M. Blagojević and F. W. Hehl, Gauge Theories of Gravitation: A Reader with Commentaries (World Scientific, Singapore, 2013).

[3] A. D. I. Latorre, G. J. Olmo, and M. Ronco, Observable traces of non-metricity: New constraints on metric-affine gravity, Phys. Lett. B 780, 294 (2018).

[4] A. Conroy and T. Koivisto, The spectrum of symmetric teleparallel gravity, Eur. Phys. J. C 78, 923 (2018).
[5] E. Alvarez, J. Anero, S. Gonzalez-Martin, and R. SantosGarcia, Physical content of quadratic gravity, Eur. Phys. J. C 78, 794 (2018).

[6] D. Iosifidis, A. C. Petkou, and C. G. Tsagas, Torsion/ non-metricity duality in $\mathrm{f}(\mathrm{R})$ gravity, Gen. Relativ. Gravit. 51, 66 (2019).

[7] D. Iosifidis and T. Koivisto, Scale transformations in metricaffine geometry, Universe, arXiv:1810.12276.

[8] J. B. Jiménez, L. Heisenberg, and T.S. Koivisto, The geometrical trinity of gravity, Universe 5, 173 (2019).

[9] J. Beltrán Jiménez and A. Delhom, Ghosts in metric-affine higher order curvature gravity, Eur. Phys. J. C 79, 656 (2019). 
[10] L. Järv, M. Rünkla, M. Saal, and O. Vilson, Nonmetricity formulation of general relativity and its scalar-tensor extension, Phys. Rev. D 97, 124025 (2018).

[11] A. Delhom, V. Miralles, and A. Peñuelas, Effective interactions in Ricci-Based gravity models below the nonmetricity scale, arXiv:1907.05615.

[12] K. Shimada, K. Aoki, and K. I. Maeda, Metric-affine gravity and inflation, Phys. Rev. D 99, 104020 (2019).

[13] K. Aoki and K. Shimada, Scalar-metric-affine theories: Can we get ghost-free theories from symmetry?, Phys. Rev. D 100, 044037 (2019).

[14] K. S. Stelle, Renormalization of higher derivative quantum gravity, Phys. Rev. D 16, 953 (1977).

[15] I. G. Avramidi and A. O. Barvinsky, Asymptotic freedom in higher derivative quantum gravity, Phys. Lett. B 159, 269 (1985).

[16] A. Salam and J.A. Strathdee, Remarks on high-energy stability and renormalizability of gravity theory, Phys. Rev. D 18, 4480 (1978).

[17] E. T. Tomboulis, Unitarity in Higher Derivative Quantum Gravity, Phys. Rev. Lett. 52, 1173 (1984).

[18] R. Floreanini and R. Percacci, The renormalization group flow of the Dilaton potential, Phys. Rev. D 52, 896 (1995).

[19] P. D. Mannheim, Solution to the ghost problem in fourth order derivative theories, Found. Phys. 37, 532 (2007).

[20] D. Anselmi and M. Piva, The ultraviolet behavior of quantum gravity, J. High Energy Phys. 05 (2018) 027.

[21] D. Anselmi and M. Piva, Quantum gravity, fakeons and microcausality, J. High Energy Phys. 11 (2018) 021.

[22] J.F. Donoghue and G. Menezes, Unitarity, stability and loops of unstable ghosts, Phys. Rev. D 100, 105006 (2019).

[23] A. Salvio and A. Strumia, Agravity, J. High Energy Phys. 06 (2014) 080.

[24] M. B. Einhorn and D. R. T. Jones, Naturalness and dimensional transmutation in classically scale-invariant gravity, J. High Energy Phys. 03 (2015) 047.

[25] B. Holdom and J. Ren, QCD analogy for quantum gravity, Phys. Rev. D 93, 124030 (2016).

[26] L. Alvarez-Gaume, A. Kehagias, C. Kounnas, D. Lüst, and A. Riotto, Aspects of quadratic gravity, Fortschr. Phys. 64, 176 (2016).

[27] J. F. Donoghue, Quartic propagators, negative norms and the physical spectrum, Phys. Rev. D 96, 044007 (2017).

[28] D. M. Ghilencea, Spontaneous breaking of Weyl quadratic gravity to Einstein action and Higgs potential, J. High Energy Phys. 03 (2019) 049.

[29] E. Sezgin and P. van Nieuwenhuizen, New ghost free gravity Lagrangians with propagating torsion, Phys. Rev. D 21, 3269 (1980).

[30] E. Sezgin, Class of ghost free gravity Lagrangians with massive or massless propagating torsion, Phys. Rev. D 24, 1677 (1981).
[31] G. K. Karananas, The particle spectrum of parity-violating Poincaré gravitational theory, Classical Quantum Gravity 32, 055012 (2015).

[32] M. Blagojević and B. Cvetković, General Poincaré gauge theory: Hamiltonian structure and particle spectrum, Phys. Rev. D 98, 024014 (2018).

[33] Y. C. Lin, M. P. Hobson, and A. N. Lasenby, Ghost and tachyon free Poincaré gauge theories: A systematic approach, Phys. Rev. D 99, 064001 (2019).

[34] P. Baikov, M. Hayashi, N. Nelipa, and S. Ostapchenko, Ghost- and Tachyon-free gauge-invariant, Poincarè, affine and projective Lagrangians, Gen. Relativ. Gravit. 24, 867 (1992).

[35] R. Percacci, The Higgs phenomenon in quantum gravity, Nucl. Phys. B353, 271 (1991).

[36] I. Kirsch, A Higgs mechanism for gravity, Phys. Rev. D 72, 024001 (2005).

[37] M. Leclerc, The Higgs sector of gravitational gauge theories, Ann. Phys. (Amsterdam) 321, 708 (2006).

[38] R. Percacci, Gravity from a particle physicists' perspective, Proc. Sci., ISFTG2009 (2009) 011 [arXiv:0910.5167]

[39] S. Gielen, R. de León Ardón, and R. Percacci, Gravity with more or less gauging, Classical Quantum Gravity 35, 195009 (2018).

[40] O. V. Babourova and B. N. Frolov, Gauss-Bonnet type identity in Weyl-Cartan space, Int. J. Mod. Phys. A 12, 3665 (1997).

[41] B. Janssen and A. Jiménez-Cano, On the topological character of metric-affine Lovelock Lagrangians in critical dimensions, Phys. Lett. B 798, 134996 (2019).

[42] D. Iosifidis, Linear transformations on affine-connections, arXiv:1911.04535.

[43] R. J. Rivers, Lagrangian theory for neutral massive spin-2 fields, Nuovo Cimento 34, 386 (1964).

[44] K. J. Barnes, Lagrangian theory for the second rank tensor field, J. Math. Phys. (N.Y.) 6, 788 (1965).

[45] A. Aurilia and H. Umezawa, Theory of high-spin fields, Phys. Rev. 182, 1682 (1969).

[46] D. E. Neville, Gravity theories with propagating torsion, Phys. Rev. D 21, 867 (1980).

[47] E. L. Mendonca and R. Schimidt Bittencourt, Unitarity of Singh-Hagen model in $D$ dimensions, arXiv:1902.05118.

[48] F. A. Berends, J. W. van Holten, P. van Nieuwenhuizen, and B. de Wit, On field theory for massive and massless spin 5/2 particles, Nucl. Phys. B154, 261 (1979).

[49] P. Baekler, N. Boulanger, and F. W. Hehl, Linear connections with propagating spin-3 field in gravity, Phys. Rev. D 74, 125009 (2006).

[50] R. Schimidt Bittencourt, Unitariedade de modelos de spin-3, Master thesis, Sao Paulo State University, https://repositorio .unesp.br/handle/11449/190789?locale-attribute=en.

[51] R. Floreanini and R. Percacci, Average effective potential for the conformal factor, Nucl. Phys. B436, 141 (1995). 